\title{
Downlink Error Rates of Half-duplex Users in Full-duplex Networks over a Laplacian Inter-User Interference Limited and EGK fading
}

\author{
Hamza Soury, Student Member, IEEE, Hesham ElSawy, Member, IEEE, \\ and Mohamed-Slim Alouini, Fellow, IEEE
}

\begin{abstract}
This paper develops a mathematical framework to study downlink error rates and throughput for half-duplex (HD) terminals served by a full-duplex (FD) base station (BS). The developed model is used to motivate long term pairing for users that have non-line of sight (NLOS) interfering link. Consequently, we study the interferer limited problem that appears between NLOS HD users-pair that are scheduled on the same FDchannel. The distribution of the interference is first characterized via its distribution function, which is derived in closed form. Then, a comprehensive performance assessment for the proposed pairing scheme is provided by assuming Extended Generalized- $\mathcal{K}$ (EGK) fading for the downlink and studying different modulation schemes. To this end, a unified closed form expression for the average symbol error rate is derived. Furthermore, we show the effective downlink throughput gain harvested by the pairing NLOS users as a function of the average signal-to-interferenceratio when compared to an idealized HD scenario with neither interference nor noise. Finally, we show the minimum required channel gain pairing threshold to harvest downlink throughput via the FD operation when compared to the HD case for each modulation scheme.
\end{abstract}

Index Terms-Interference limited, complex Gaussian, characteristic function, Laplace distribution, symbol error rate, EGK fading

\section{INTRODUCTION}

In-band full-duplex (FD) communication is introduced as a promising technology that would provide several benefits to cellular networks. Compared to its half-duplex (HD) counterpart, FD communication has the potential to improve spectrum utilization, increase link capacity, enhance physical layer security, and reduce relaying latency [1]. The aforementioned benefits brought by FD communication are all derived from its ability to simultaneously transmit and receive within the same frequency band. The ability of simultaneous transmission and reception for FD transceivers emerges from recent advances in radio frequency circuit design that enables sufficient self-interference (SI) cancellation [2], [3], and hence, eliminates the necessity for transmission/reception orthogonalization employed by HD transceivers [1], [4]-[6]. To reap the aforementioned benefits of FD communication, FD enabled transceivers are required at both sides of the

The authors are with King Abdullah University of Science and Technology (KAUST), Thuwal, Makkah Province, Kingdom of Saudi Arabia. The authors are members of the KAUST Strategic Research Initiative (SRI) in Uncertainty Quantification. E-mail: \{soury.hamza, hesham.elsawy, slim.alouini\}@kaust.edu.sa.

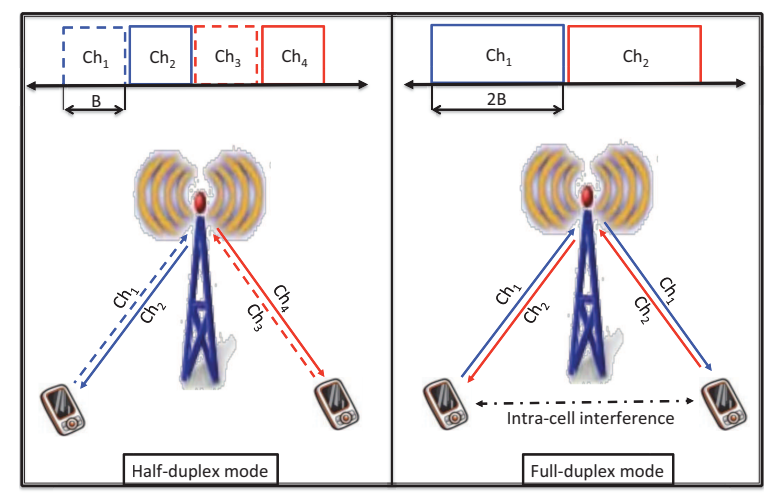

Fig. 1: Channel assignment in the 3NT FD and HD schemes.

communication link, which is hard to realize in the context of cellular networks.

Cellular networks operators can only enforce FD upgrade in their networks from the base stations' (BS) side and do not have direct access to upgrade the users equipment (UE) side. It has been shown in [7] that the residual self interference amplitude can be degraded significantly from $-20 \mathrm{~dB}$ to $-60 \mathrm{~dB}$ for frequencies between $2.35 \mathrm{GHz}$ and $2.45 \mathrm{GHz}$. However, implementing SI cancellation at the UE can be expensive in terms of complexity, power consumption, and/or terminal price. Hence, backward compatibility between FD BSs and HD UEs is required especially at the early FD rollout phase. In this context, the 3-node topology (3NT) is proposed to serve HD users via FD BSs and yet benefit from FD communication [8], [9]. In the 3NT, the BS groups the UEs into pairs and simultaneously reuses the uplink channel of one UE in the downlink direction of the other UE in each pair, as shown in Fig. 1. Compared to the HD case, the 3NT requires half the number of channels to serve the same number of users, and hence, the bandwidth (BW) occupied by each channel can be doubled [10].

Doubling the channel BW directly implies doubling the transmission rate, but not necessarily doubling the throughput. This is because the BW improvement, offered by the 3NT, comes at the expense of creating SI at the BS side and inter-user interference at the UE side, which impose decoding errors. The BS exactly knows the interfering codeword and can accurately estimate the SI channel, and hence, SI can be sufficiently suppressed using SI cancellation techniques [1], 
[4]-[6]. However, the UE does not have information about the signal being transmitted by the interfering UE, and hence, interference cancellation is not always viable. The authors in [8] proposed an interference alignment technique to eliminate the inter-user interference between the UEs pair coupled on the same channel. The authors in [11] mitigate the interuser interference via an optimal channel allocation and power control that maximizes the system performance. In addition, in [12], [13] the authors investigate the user scheduling and power allocation by maximizing the system sum rate. However, the algorithms in [8], [11]-[13] require the instantaneous mutual channel state information between UEs, which is hard to estimate and/or communicate. A more practical way to mitigate the inter-user interference is to pair interfering users in the $3 \mathrm{NT}$ based on their statistical mutual channel conditions, which requires statistical studies for the inter-user interference problem.

\section{A. Motivation and objectives}

This paper proposes pairing users according to their long term statistical channel conditions ${ }^{1}$ Such user pairing criterion only requires the statistical information about the mutual channel gains between the users rather than the instantaneous channel gains, which will relief the computational complexity of the scheduling procedure and reduce signaling overhead. Particularly, we propose pairing users that have non-line of sight (NLOS) mutual interfering links. To support such pairing methodology, a comprehensive performance assessment is provided. First, assuming Rayleigh fading on the interference link $k^{2}$, the inter-user interference is characterized by deriving closed form expressions for its probability density function (PDF), cumulative density function (CDF), and characteristic function (CHF). Then, the error rate performance is assessed for several downlink fading channels and modulation schemes. Specifically, the interference distribution is exploited to characterize the error rates for binary phase shift keying (BPSK), pulse amplitude modulation (PAM), quadrature amplitude modulation (QAM), and $M$ phase shift keying (MPSK) modulations over Extended Generalized- $\mathcal{K}$ (EGK) fading channel [15].A unified closed-from expression for the average symbol error rate (SER) is derived. To this end, the throughput of the proposed pairing scheme is characterized and compared to its HD counterpart. Finally, we provide insights for the modulation scheme selection based on the average SIR. To the best of our knowledge, our paper is the first to advocate long term pairing schemes for HD users served by FD BS and provide comprehensive performance assessment.

\section{B. Summary of Contributions}

It ought to be mentioned that outage probability and achievable rate analysis for the $3 \mathrm{NT}$ cellular networks are conducted in [16]-[19]. However, different from [16]-[19], this paper is the first to obtain a closed form expression for the inter-user

\footnotetext{
${ }^{1}$ This work is presented in part in $|14|$.

${ }^{2}$ Rayleigh fading environment reflects NLOS links in rich scattering environment.
}

interference distribution, derive exact error rates for different modulation schemes, present a unified expression for error rates for different modulation schemes, and show the explicit effect of inter-user interference on the throughput of each modulation scheme. From the mathematical perspective, this paper offers an explicit form of the average probability of error of the above system under an EGK fading channel [15]. The EGK is selected on the useful link for its flexibility to capture different fading environments as special cases [20], including Rayleigh, Nakagami- $m$, Generalized Nakagami-m (GNM), Gamma, Weibull, and others as can be seen in Table III.

The remaining of the paper is organized as follows. Section II presents the system model and assumptions. The distribution of the inter-user interference is derived in Section III. The conditional error rates are analyzed in Section IV, i.e., conditioning over the useful link fading. Then, the average error rates are studied in Section V. Section VI focuses on the throughput performance and Section VII presents and discusses selected numerical results. Finally, Section VIII concludes the paper.

\section{SYSTEM MODEL}

This paper explicitly focuses on the inter-user interference problem for NLOS HD users paired on the same channel imposed in the 3NT shown in Fig. 1. Therefore, we consider a single cell scenario with an FD BS and two NLOS HD UEs. Without loss in generality, we assume that spectrum can be either divided into four non-overlapping channels that are assigned in HD mode or two non-overlapping channel that are assigned using the 3NT FD mode, as shown in Fig. 1 In the HD case, each user is assigned an interference free bandwidth (BW) $B \mathrm{~Hz}$ in each of the uplink and downlink directions. On the other hand, the 3NT FD mode doubles the BW to $2 B$ in each of the uplink and downlink directions at the expense of SI in the uplink and inter-user interference in the downlink. Without loss of generality, we focus on the downlink performance of one of the UEs. The complex baseband received signal at the test UE can be expressed as

$$
r=\sqrt{P_{d} \mathcal{A}_{d}} h s+\sqrt{P_{u} \mathcal{A}_{u}} g x+n,
$$

where $P_{d}$ is the downlink transmit power, $\mathcal{A}_{d}$ is a constant that captures downlink large-scale power attenuation, $s$ is the intended symbol drawn from a unit power constellation, $h$ is the intended channel with unit-mean EGK distributed power gain, $P_{u}$ is the power transmitted by the interfering UE, $\mathcal{A}_{u}$ is a constant that captures large-scale interference power attenuation, $x$ is the inter-user interfering Gaussian symbol, $g$ is the unit variance circularly symmetric complex Gaussian mutual channel gain between the UEs and $n$ is the additive white noise. The random variables $h, s, g, x$, and $n$ are assumed to be independent.

Remark 1The circularly symmetric Gaussian distribution of $g$ models a severe Rayleigh fading scenario, which occurs in NLOS scattering rich environments. The Rayleigh fading assumption on the interference channel is a direct consequence of the proposed NLOS pairing criterion, which can be realized 
by multi-user diversity in which the BS would pair only NLOS UEs on the same uplink/downlink channels. It is worth noting that the analysis in [16]-[19] is based on the Rayleigh fading assumption on both the interfering and the useful links for analytical tractability.

Remark 2Unlike $|g|$, which is assumed Rayleigh by virtue of the NLOS user pairing and multi-user diversity, the proposed pairing scheme do not put any constraint on the downlink link channel fading. Hence, $|h|$ is modeled using the general EGK fading distribution to provide comprehensive performance assessment for the NLOS pairing criterion under diverse types of fading channels that can appear between the user and the serving BS.

Remark 3 The Gaussian signaling abstraction for the interfering symbols is used in the literature and is shown to have negligible effect on the error rate performance [21], [22]. Furthermore, gaussian signals provide accurate approximation for the interference generated from a single node transmitting on a faded channel [21]. This assumption is also validated in Section VI of this paper.

Remark 4 The intended signal power is $P_{d}$ and the interfering signal power is $P_{u}$ because we study the downlink transmission in which the intra-mode interference comes from an uplink UE, as shown in Fig. 1

For analytical tractability, we assume that the inter-user interference dominates the noise, and hence, the noise term is ignored in the analysis. The accuracy of this approximation is validated in the results section with realistic noise power. Ignoring the white noise, the received signal $r$ can be rewritten as

$$
r \approx \sqrt{P_{d} \mathcal{A}_{d}} h s+\sqrt{P_{u} \mathcal{A}_{u}} g x .
$$

The received signal $r$ is a complex valued random variable with real and imaginary components, which are represented as $r=\mathfrak{R}(r)+j \mathfrak{I}(r)$, where $\mathfrak{R}(\cdot)$ and $\mathfrak{I}(\cdot)$ denote the real and imaginary parts of a complex variable, respectively, and $j=\sqrt{-1}$ is the imaginary unit. To study (2), we first focus on the interference term, which can be considered as an additive complex perturbation for the useful symbol $s$. In the next section, we study the normalized inter-user interference, denoted as $Z=g x$. The normalized inter-user interference $Z$ is characterized by closed form expressions for its PDF and CDF.

\section{Distribution OF THE INTER-USER INTERFERENCE}

In what follows subscripts $r$ and $i$ denote the real and imaginary parts, respectively.

Since $g$ and $x$ are both zero-mean unit-variance complex Gaussian random variables, their real part and imaginary part are independent Gaussian random variables with zero-mean and variance $\frac{1}{2}$. The inter-user Interference $Z$ is represented as

$$
\begin{aligned}
Z & =g x \\
& =(\mathfrak{R}(g) \mathfrak{R}(x)-\Im(g) \Im(x))+j(\mathfrak{I}(g) \mathfrak{I}(x)+\mathfrak{I}(g) \mathfrak{R}(x)) .
\end{aligned}
$$

The distribution of $Z$ can be obtained using the relations between the CHF, the PDF, and the CDF. To benefit from these relations, the CHF, denoted by $\varphi_{Z}\left(t_{1}, t_{2}\right)$, should be derived first. Thus, Lemma 1 gives the CHF of the inter-user interference.

Lemma 1 The inter-user interference from a UE transmitting Gaussian signals over a Rayleigh faded channel has the following characteristic function

$$
\varphi_{Z}\left(t_{1}, t_{2}\right)=\frac{4}{4+t_{1}^{2}+t_{2}^{2}}=\frac{1}{1+\frac{|\mathbf{t}|^{2}}{4}},
$$

where the vector $\mathbf{t}=\left(t_{1}, t_{2}\right)$.

Proof: See Appendix A

Remark 5 The expression in (4) is a special case of the bivariate Laplace distribution [23]. Furthermore, the CHF of $Z$ is independent of the angle between the real and imaginary components. Consequently, (4) shows that $Z$ follows a circularly symmetric complex Laplace distribution.

From the CHF of $Z$ given in Lemma 1 , the CDF of $Z$ is given in the following theorem.

Theorem 1. The inter-user interference from a UE transmitting Gaussian signals over a Rayleigh faded channel has the following $C D F$

$$
\begin{aligned}
& F_{Z}\left(z_{r}, z_{i}\right)=1-\frac{1}{2}\left(e^{-2 z_{r}}+e^{-2 z_{i}}\right) \\
& +\frac{1}{4 \pi} \mathrm{G}_{1,0,2,1,2,1}^{0,1,0,2,0,2}\left[\frac{1}{z_{r}^{2}}, \frac{1}{z_{i}^{2}} \mid \begin{array}{c|c|c}
0 & \frac{1}{2}, 1 & \frac{1}{2}, 1 \\
0 & 0
\end{array}\right],
\end{aligned}
$$

where G. $\because[., \cdot]$ is the bivariate Meijer's $G$ function (BMGF), an extension of the Meijer's G function, that is defined in [24], where an optimal code in Mathematica was provided too.

Proof: See Appendix B.

It is important to note that the PDF of the product of two independent complex Gaussian random variables has been studied before in [25], [26]. However, the expression in Theorem 1 presents a novelty, since this is the first time the CDF of the product of two independent complex Gaussian random variables is described in closed form. Consequently, the PDF of the normalized inter-user interference (i.e., $Z$ ) is given in the following corollary.

Corollary 1.1 The inter-user interference from a UE transmitting Gaussian signals over a Rayleigh faded channel has the following PDF

$$
f_{Z}\left(z_{r}, z_{i}\right)=\frac{2}{\pi} K_{0}\left(2 \sqrt{z_{r}^{2}+z_{i}^{2}}\right),
$$

where $K_{0}(\cdot)$ is the 0 -th order modified Bessel function of the second kind [27, Eq. (9.6.21)].

Proof: See Appendix C

Remark 6 The PDF expression in (6) clearly shows that $Z$ does not have independent real and imaginary components because the joint PDF in (6) cannot be expressed as the product of two marginal Laplace PDFs. 
Remark 7 The problem of decoding in the presence of additive Laplacian perturbation (i.e., noise or interference) has been widely addressed in the literature [28]-[32]. However, a common assumption in this literature is that the Laplacian perturbation has independent real and imaginary components. Hence, the work presented in this paper contributes to the literature by modeling the case with dependent real and imaginary components. Furthermore, this paper rigorously derive the Laplacian distribution of the interference from the system model rather than assuming a Laplacian perturbation.

Exploiting the interference characterization in this section, the error rate performance is analyzed in the next sections.

\section{Conditional ERror Rates}

This section characterizes the downlink decoding errors that may occur due to the Laplacian inter-user interference with the PDF shown in (6). In particular, we evaluate the probability of BPSK, PAM, QAM, and MPSK. At first, the conditional SER (i.e., conditioning on the intended channel gain) as a function of the signal-to-interference ratio (SIR), denoted as $\gamma=\frac{P_{d} \mathcal{A}_{d}|h|^{2}}{P_{u} \mathcal{A}_{u}}$, is investigated. The conditional error rate analysis can also be considered as the error rate over unfaded downlink channe 3 By the end of this section, a unified expression of the conditional SER is presented.

For the sake of organized presentation, we classify the studied modulation schemes into two sets, namely, $(i)$ one dimensional constellations (1D) (i.e. BPSK and M-PAM) and (ii) two dimensional constellations $(2 D)$ (i.e. M-QAM and MPSK). Then, the analysis of each class is presented in a separate section.

\section{A. Error Rate for $1 D$ Constellations}

The conditional bit error rate (BER) for BPSK is giving is the following Theorem.

Theorem 2. The conditional downlink bit error rate in $3 N T$ with Laplacian inter-user interference and maximum likelihood detector for the BPSK modulation is given by

$$
P_{B P S K}(e \mid \gamma)=\frac{1}{2} e^{-2 \sqrt{\gamma}} .
$$

Proof: Since the BPSK transmits real symbols $s= \pm E_{s}$, the conditional PDF of the received signal $r$ is obtained from (6) as $f_{r}(r)=e^{-2\left|r \mp E_{s}\right|}$. Moreover, the optimal decision is obtained by minimizing the distance $d\left(r, \pm E_{s}\right)$. Given that the BPSK symbols are equiprobable, the probability of error can be computed as $\operatorname{Pr}(e \mid \gamma)=\int_{0}^{\infty} f_{r}\left(r \mid S=-E_{s}\right) d r$. Then (7) is obtained by setting $E_{s}=1$ due to the assumed unit energy constellation.

Based on Theorem 2, the probability of error of an MPAM modulation is characterized in the following corollary

Corollary 2.1 The conditional downlink SER in 3NT with Laplacian inter-user interference and maximum likelihood detector for the MPAM modulation is given by

$$
P_{P A M}(e \mid \gamma)=\left(1-\frac{1}{M}\right) e^{-2 \sqrt{\frac{3 \gamma}{M^{2}-1}}},
$$

\footnotetext{
${ }^{3}$ An important application for unfaded channels appears in the context of massive MIMO systems due to the channel hardening effect [33].
}

where the exponent argument $\sqrt{\frac{3 \gamma}{M^{2}-1}}$ is obtained by calculating the average energy per symbol for MPAM.

Proof: The corollary directly follows from the BER of a BPSK modulation as $P_{P A M}(e)=2\left(1-\frac{1}{M}\right) P_{B P S K}(e)$. Hence, the conditional SER of MPAM modulation can be deduced from (7).

\section{B. Error Rate for $2 D$ Constellations}

In this section we consider $2 \mathrm{D}$ constellations formed by in-phase and quadrature phase components. Particularly, we focus on the M-QAM and the MPSK constellations. While the exact closed-from expressions of the SERs for the MQAM and the MPSK schemes are derived, we also present simplified approximations in order to present a unified error rate expression for all of the considered modulation schemes.

1) SER for Rectangular QAM: A rectangular $M$-QAM is formed by one in-phase $M_{I}$-PAM and orthogonal quadrature phase $M_{Q}$-PAM, which means that $M=M_{I} M_{Q}$. The rectangular QAM modulation is a generalization of the square QAM that can cover more signal constellations. Therefore, the SER of rectangular QAM modulation is given in the following theorem.

Theorem 3. The conditional downlink SER in $3 N T$ with Laplacian inter-user interference and maximum likelihood detector for the rectangular QAM modulation is given by

$$
\begin{aligned}
& P_{Q A M}(e \mid \gamma)=\rho\left(M_{I}\right) e^{-2 \frac{\sqrt{3 \gamma}}{d}}+\rho\left(M_{Q}\right) e^{-2 \tau \frac{\sqrt{3 \gamma}}{d}} \\
& \quad-\frac{1}{\pi} \rho\left(M_{I}\right) \rho\left(M_{Q}\right) \mathrm{G}_{1,0,2,1,2,1}^{0,1,0,2,0,2}\left[\frac{d^{2}}{3 \gamma}, \frac{d^{2}}{3 \tau^{2} \gamma} \mid \begin{array}{c|c|c}
0 & \frac{1}{2}, 1 & \frac{1}{2}, 1 \\
- & 0 & 0
\end{array}\right],
\end{aligned}
$$

where $d=\sqrt{\left(M_{I}^{2}-1\right)+\tau^{2}\left(M_{Q}^{2}-1\right)}, \tau$ is the in-phaseto-quadrature decision distance ratio defined as $\tau=\frac{d_{Q}}{d_{I}}$, and $\rho(x)=1-\frac{1}{x}$. The terms $d_{I}$ and $d_{Q}$ represent the in-phase and quadrature phase decision distance, respectively.

Proof: See Appendix D

A special case of Theorem 3 is the square QAM, where $M_{I}=M_{Q}=\sqrt{M}$ and $\tau=1$. Using the double MellinBarnes contour integral of the BMGF [34] and the expression of the SER in Theorem 3, the SER of square QAM can be reduced to

$$
\begin{aligned}
& P_{S Q A M}(e \mid \gamma)=2 \rho(\sqrt{M}) e^{-\sqrt{\frac{6 \gamma}{M-1}}} \\
& \quad-\frac{1}{\pi} \rho(\sqrt{M})^{2} \mathrm{G}_{2,1,1,2,1,0}^{0,2,2,0,0}\left[\frac{2(M-1)}{3 \gamma}, 1 \mid \begin{array}{c|c|c|c}
\frac{1}{2}, 1 & 1 & 0 \\
0 & \frac{1}{2}, 0 & -
\end{array}\right] .
\end{aligned}
$$

The error rate for the rectangular M-QAM modulation given in Theorem 3 is expressed in terms of the BMGF, which imposes high computational complexity. Therefore, we propose the following simplifying approximation.

Proposition 1 The conditional downlink SER in 3NT with Laplacian inter-user interference and maximum likelihood 
detector for the rectangular QAM modulation can be approximated by

$$
\begin{aligned}
P_{Q A M}^{A}(e \mid \gamma) & =\rho\left(M_{I}\right) e^{-2 \frac{\sqrt{3 \gamma}}{d}}+\rho\left(M_{Q}\right) e^{-2 \tau \frac{\sqrt{3 \gamma}}{d}} \\
& -\rho\left(M_{I}\right) \rho\left(M_{Q}\right) e^{-2(1+\tau) \frac{\sqrt{3 \gamma}}{d}} .
\end{aligned}
$$

In the case of square QAM modulation, the SER approximation reduces to

$$
P_{S Q A M}^{A}(e \mid \gamma)=2 \rho(\sqrt{M}) e^{-\sqrt{\frac{6 \gamma}{M-1}}}-\rho(\sqrt{M})^{2} e^{-2 \sqrt{\frac{6 \gamma}{M-1}}} .
$$

Proof: The approximation is obtained by considering a QAM modulation formed by two orthogonal (approximated as independent in presence of Laplace interference) PAMs. Thereby, a correct detection of the $M$-QAM appears only for a correct detection in the $M_{I}$-PAM and $M_{Q}$-PAM. Consequently, the probability of error of $M$-QAM may be approximated as

$$
\begin{aligned}
& P_{Q A M}^{A}(e)=1-\left(1-P_{P A M_{I}}(e)\right)\left(1-P_{P A M_{Q}}(e)\right) \\
& \quad=P_{P A M_{I}}(e)+P_{P A M_{Q}}(e)-P_{P A M_{I}}(e) P_{P A M_{Q}}(e) .
\end{aligned}
$$

Then (11) is obtained by substituting (8) in (13).

2) SER of MPSK: This section considers the case where the transmitted signal $s$ is modulated via an MPSK scheme with $M$ being a power of 2 and $M \geq 4$, the BER of the BPSK case, i.e. $M=2$, was derived in Theorem 7 , The MPSK symbols are distributed uniformly over the unit circle and all symbols are equiprobable. Unlike the previous modulation schemes, the decision regions for the MPSK symbols are not known a priori and have to be characterized for each complex perturbation. Let $I$ and $Q$ be the normalized in-phase and quadrature phase components of the received signal, then the likelihood function and decision criterion under the complex Laplacian perturbation given in (6) is characterized in the following lemma.

Lemma 2 The likelihood function for the $k$-th symbol $s_{k}$ in an MPSK constellation under the complex Laplacian perturbation given in (6) can be expressed as

$$
\ell_{k}=R \cos \left(\psi-\varphi_{k}\right),
$$

where $R=\sqrt{I^{2}+Q^{2}}$ and $\psi=\arctan \left(\frac{Q}{I}\right)$. Consequently, the decision criterion for the MPSK received symbols can be defined by selecting the symbol $s_{k}$ which minimizes $\left|\psi-\varphi_{k}\right|$, where $\varphi_{k}=\frac{2 k \pi}{M}$.

Proof: According to the PDF of $Z$ in (6), the likelihood function can be written as

$$
\ell_{k}=\frac{2 \gamma}{\pi} K_{0}\left(2 \sqrt{\gamma} \sqrt{\left(I-\cos \varphi_{k}\right)^{2}+\left(Q-\sin \varphi_{k}\right)^{2}}\right),
$$

where

$$
\left\{\begin{array}{l}
I=s_{r}^{(k)}+\frac{z_{r}}{\sqrt{\gamma}}=\cos \varphi_{k}+\frac{z_{r}}{\sqrt{\gamma}} \\
Q=s_{i}^{(k)}+\frac{z_{i}}{\sqrt{\gamma}}=\sin \varphi_{k}+\frac{z_{i}}{\sqrt{\gamma}} .
\end{array}\right.
$$

From 27, Eqs. (9.6.27) \& (9.6.1)], we know that $\frac{\mathrm{d} K_{0}(x)}{\mathrm{d} x}=$ $-K_{1}(x)$ and that $K_{1}(x)$ is positive for $x>0, K_{1}(\cdot)$ is the 1-st order modified Bessel function of the second kind. Hence,
$K_{0}(\cdot)$ is a monotonically decreasing function. Consequently, the likelihood function $\ell_{k}$ is maximized by minimizing the Bessel function argument, i.e., minimizing the distance $(I-$ $\left.\cos \varphi_{k}\right)^{2}+\left(Q-\sin \varphi_{k}\right)^{2}$. Using some algebraic simplification, $\ell_{k}$ can be expressed as follows

$$
\begin{aligned}
\ell_{k} & =I \cos \varphi_{k}+Q \sin \varphi_{k} \\
& =R\left(\cos \psi \cos \varphi_{k}+\sin \psi \sin \varphi_{k}\right),
\end{aligned}
$$

which gives 14 and completes the proof.

An illustrative example for the MPSK decision regions, with $M=8$, that maximizes the likelihood function $\ell_{k}$ is shown in Fig 2. Following similar methodology to [35], the decision regions are obtained by defining the two coordinates rotations $\left(X_{1}, Y_{1}\right)$, with rotation angle $\phi-\theta$, and $\left(X_{2}, Y_{2}\right)$, with rotation angle $\phi+\theta$, where $\phi$ is the symbol phase and $\theta=\frac{\pi}{M}$. The circular symmetry of the interference PDF in (6) implies that all symbols have similar error probability. Hence, the total error probability can be deduced by considering a generic single symbol, with angle $\varphi$ lies between 0 and $\frac{\pi}{2}$, and the error probability can be explicitly expressed in terms of $Y_{1}$ and $Y_{2}$ as

$$
P e(\gamma, \phi)=\operatorname{Pr}\left[Y_{1} \geq 0\right]+\operatorname{Pr}\left[Y_{2} \leq 0\right]-\operatorname{Pr}\left[Y_{1} \geq 0, Y_{2} \leq 0\right] .
$$

where $Y_{1}$ and $Y_{2}$ are obtained from the aforementioned rotation as

$$
\left[\begin{array}{l}
Y_{1} \\
Y_{2}
\end{array}\right]=\left[\begin{array}{ll}
-\sin (\phi-\theta) & \cos (\phi-\theta) \\
-\sin (\phi+\theta) & \cos (\phi+\theta)
\end{array}\right]\left[\begin{array}{l}
I \\
Q
\end{array}\right] .
$$

Based on (17) and 18), the SER for the MPSK constellation is characterized in the following theorem

Theorem 4. The probability of error detection of one symbol with phase $\varphi$ using maximum likelihood detector is given as follows

$$
\begin{aligned}
& P_{e}(\gamma, \varphi)=e^{-2 \delta \sqrt{\gamma}} \\
& -\frac{\delta}{2 \sqrt{\pi}} \mathrm{G}_{1,1,1,1,2,0}^{0,1,1,0,0}\left[\delta^{2}, \frac{4 \delta^{2}\left(1-\delta^{2}\right)}{\gamma\left(\cos ^{2} \varphi-\delta^{2}\right)} \mid \begin{array}{c|c|c}
\frac{1}{2} & \frac{1}{2} & 1,0 \\
-\frac{1}{2} & 0 & -
\end{array}\right],
\end{aligned}
$$

where $\delta=\sin \left(\frac{\pi}{M}\right)$ and $\varphi=\frac{2 k \pi}{M}$.

Proof: See Appendix E

Once the probability of error of a single symbol is obtained, the total probability of error for MPSK can be deduced directly in Corollary 4.1 .

Corollary 4.1 The conditional downlink SER in 3NT with Laplacian inter-user interference and maximum likelihood detector for the MPSK modulation is given by

$$
\begin{aligned}
& P_{M P S K}(e \mid \gamma)=e^{-2 \delta \sqrt{\gamma}}-\frac{2 \delta}{M \sqrt{\pi}} \\
& \times \sum_{k=0}^{\frac{M}{4}-1} \mathrm{G}_{1,1,1,1,2,0}^{0,1,1,0,0,2}\left[\delta^{2}, \frac{4 \delta^{2}\left(1-\delta^{2}\right)}{\gamma\left(\cos \left(\frac{2 k \pi}{M}\right)^{2}-\delta^{2}\right)} \mid \begin{array}{c|c|c|c}
\frac{1}{2} & \frac{1}{2} & 1,0 \\
-\frac{1}{2} & 0 & -
\end{array}\right] .
\end{aligned}
$$

Proof: The total probability of error of the MPSK modulation can be computed as the average of the probability of 


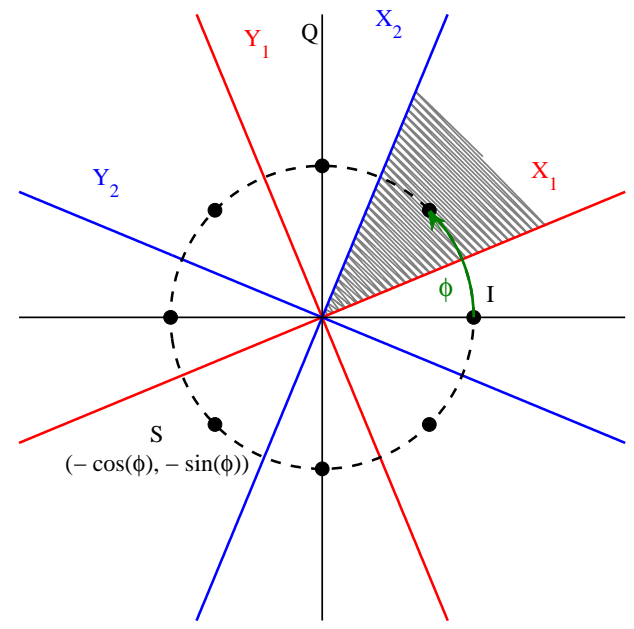

Fig. 2: Decision regions using maximum likelihood detector for 8-PSK.

error over all the symbols. Since the perturbation PDF in (6) is circular symmetric, we can focus our study to the region between 0 and $\frac{\pi}{2}$ and the total SER of the MPSK can be written as

$$
P_{M P S K}(e \mid \gamma)=\frac{4}{M} \sum_{k=0}^{\frac{M}{4}-1} P_{e}\left(\gamma, \varphi_{k}\right)=\frac{4}{M} \sum_{k=0}^{\frac{M}{4}-1} P_{e}\left(\gamma, \frac{2 k \pi}{M}\right) .
$$

Replacing (19) in 21), Corollary 4.1 is obtained.

Similar to the MQAM scheme, the SER of MPSK is expressed in terms of the BMGF, which imposes high computational complexity. Hence, a simplifying approximation for the SER in MPSK modulation is given in the following proposition.

Proposition 2 The conditional downlink SER in 3NT with Laplacian inter-user interference for the MPSK modulation can be approximated by

$$
\begin{aligned}
& P_{M P S K}^{A}(e \mid \gamma)=e^{-2 \delta \sqrt{\gamma}}+\frac{2 \tan \theta}{M(1+\tan \theta)} e^{-2 \sqrt{\gamma}} \\
& +\frac{1}{M} \sum_{k=1}^{\frac{M}{4}-1} \frac{\sin 2 \theta}{\cos 2 \theta+\sin \frac{4 k \pi}{M}} e^{-2 \sqrt{2 \gamma} \sin \left(\frac{2 k \pi}{M}+\frac{\pi}{4}\right)} .
\end{aligned}
$$

Proof: The exact expressions for $\operatorname{Pr}\left[Y_{1} \geq 0\right]$ and $\operatorname{Pr}\left[Y_{2} \leq 0\right]$ are already in the exponential form, and hence, no approximation is required for these terms. On the other hand, the BMGF appears in $\operatorname{Pr}\left[Y_{1} \geq 0, Y_{2} \leq 0\right]$, which can be approximated assuming independent interference components as in [28, Eq. (30)]. It is worth noting that (22) is based on approximating only the term $\operatorname{Pr}\left[Y_{1} \geq 0, Y_{2} \leq 0\right]$ with a similar expression to [28, Eq. (30)], while the two other terms are derived for the first time in this paper using the interference

\begin{tabular}{|c|c|c|c|c|}
\hline \multirow{2}{*}{$\begin{array}{c}\text { Modulation } \\
\text { Scheme }\end{array}$} & \multicolumn{4}{|c|}{ Modulation Specific Parameters } \\
\hline & $n$ & $k$ & $a_{k}$ & $b_{k}$ \\
\hline BPSK & 1 & 1 & $\frac{1}{2}$ & 1 \\
\hline PAM & 1 & 1 & $\rho(M)$ & $2 \sqrt{\frac{3}{M^{2}-1}}$ \\
\hline \multirow{2}{*}{$\begin{array}{l}\text { Approximated } \\
\text { Square-QAM }\end{array}$} & \multirow{2}{*}{2} & 1 & $2 \rho(\sqrt{M})$ & $\sqrt{\frac{6}{M-1}}$ \\
\hline & & 2 & $-\rho(\sqrt{M})^{2}$ & $2 \sqrt{\frac{6}{M-1}}$ \\
\hline \multirow{3}{*}{$\begin{array}{c}\text { Approximated } \\
\text { Rectangular-QAM }\end{array}$} & \multirow{3}{*}{3} & 1 & $\rho\left(M_{I}\right)$ & $2 \frac{\sqrt{3}}{d}$ \\
\hline & & 2 & $\rho\left(M_{Q}\right)$ & $2 \tau \frac{\sqrt{3}}{d}$ \\
\hline & & 3 & $\rho\left(M_{I}\right) \rho\left(M_{Q}\right)$ & $2(1+\tau) \frac{\sqrt{3}}{d}$ \\
\hline \multirow{3}{*}{$\begin{array}{c}\text { Approximated } \\
\text { MPSK }\end{array}$} & \multirow{3}{*}{$\frac{M}{4}+1$} & $1 \leq k \leq n-2$ & $\frac{\sin 2 \theta}{M\left(\cos 2 \theta+\sin \frac{4 k \pi}{M}\right)}$ & $2 \sqrt{2} \sin \left(\frac{2 k \pi}{M}+\frac{\pi}{4}\right)$ \\
\hline & & $n-1$ & $\frac{2 \tan \theta}{M(1+\tan \theta)}$ & 2 \\
\hline & & $n$ & 1 & $2 \delta$ \\
\hline
\end{tabular}
described in (3).
TABLE I: SER Modulation-Specific Parameters $n, a_{k}$, and $b_{k}$

\section{Unified Expression of the SER}

By virtue of the approximations given in Proposition 1 and Proposition 2, we can obtain a unified expression for the error rates in all considered modulation schemes. Particularly, the error rates derived in Theorem 2, Corollary 2.1. Proposition 1. and Proposition 2, can be expressed using the following unified expression

$$
S E R=\sum_{k=1}^{n} a_{k} e^{-b_{k} \sqrt{\gamma}},
$$

where $n, a_{k}$, and $b_{k}$ are related to the used modulation. Their possible values are summarized in Table [

\section{Average SER over EGK FADING CHANNEL}

Using the conditional error rates obtained in the previous section, the average SER $(\overline{S E R})$ is derived by averaging over the EGK fading distribution on the downlink channel, where the EGK distribution is given in [15, Eq. (2)]. The EGK distribution presents a versatile fading envelope model that generalizes many commonly used statistical models that describe signal fluctuations in the received SIR due to multipath, shadowing, or a mixture of such processes in the environment [15]. Moreover, the EGK distribution captures many known fading distributions as special cases, like Rayleigh fading, Nakagami- $m$ fading, $K$ distribution and many other distributions summarized in [36]. In the present model, assuming that $\mathbb{E}\left[|h|^{2}\right]=1$, the average $\operatorname{SIR}$ is given by $\bar{\gamma}=\mathbb{E}[\gamma]=\frac{P_{d} \mathcal{A}_{d}}{P_{u} \mathcal{A}_{y}}$, $\mathbb{E}[\cdot]$ represented the expected value a random variable. The PDF of $\gamma$ can be expressed as follows

$$
\begin{aligned}
p_{\gamma}(\gamma)= & \frac{\xi}{\Gamma\left(m_{s}\right) \Gamma(m)}\left(\frac{\beta_{s} \beta}{\bar{\gamma}}\right)^{m \xi} \gamma^{m \xi-1} \\
& \times \Gamma\left(m_{s}-m \frac{\xi}{\xi_{s}}, 0,\left(\frac{\beta_{s} \beta}{\bar{\gamma}}\right)^{m \xi} \gamma^{m \xi}, \frac{\xi}{\xi_{s}}\right),
\end{aligned}
$$

which is defined over $\gamma \in(0, \infty)$ and the parameters $m(0.5 \leq m<\infty)$ and $\xi(0 \leq \xi<\infty)$ represent the fading figure (diversity severity/order) and the fading 
shaping factor, respectively, while $m_{s}\left(0.5 \leq m_{s}<\infty\right)$ and $\xi_{s}\left(0 \leq \xi_{s}<\infty\right)$ represent the shadowing severity and the shadowing shaping factor (inhomogeneity), respectively. In addition, we define the parameters $\beta=\Gamma(m+1 / \xi) / \Gamma(m)$ and $\beta_{s}=\Gamma\left(m_{s}+1 / \xi_{s}\right) / \Gamma\left(m_{s}\right)$. In (24), $\Gamma(\cdot, \cdot, \cdot, \cdot)$ is the extended incomplete Gamma function defined in [37, Eq. (6.2)] as

$$
\Gamma(a, x, b, \beta)=\int_{x}^{\infty} r^{a-1} \exp \left(-r-b r^{-\beta}\right) d r,
$$

where $a, \beta, b \in \mathbb{C}$ and $x \in \mathbb{R}^{+}$.

To simplify the computation, the identities [37, Eq. (6.22)] and [38. Eqs. (2.1.4), (2.1.5) and (2.1.11)] are used to express 24) in terms of the Fox's $\mathrm{H}$ function, as

$$
p_{\gamma}(\gamma)=\frac{1}{\Gamma\left(m_{s}\right) \Gamma(m) \gamma} \mathrm{H}_{0,2}^{2,0}\left[\frac{\beta_{s} \beta}{\bar{\gamma}} \gamma \mid \underset{\left(m_{s}, \frac{1}{\xi_{s}}\right),\left(m, \frac{1}{\xi}\right)}{-},\right.
$$

where $\mathrm{H}_{p, q}^{m, n}[\cdot]$ denotes the Fox's $\mathrm{H}$ function (FHF) whose definition is given by [38. Eq. (1.1.1)] and [39] as

$$
\begin{aligned}
& \mathrm{H}_{p, q}^{m, n} {\left[z \mid \begin{array}{l}
\left(a_{i}, \alpha_{i}\right)_{1, p} \\
\left(b_{j}, \beta_{j}\right)_{1, q}
\end{array}\right]=} \\
& \frac{1}{2 \pi i} \int_{\mathcal{C}} \frac{\prod_{j=1}^{m} \Gamma\left(b_{j}+\beta_{j} s\right) \prod_{i=1}^{n} \Gamma\left(1-a_{i}-\alpha_{i} s\right)}{\prod_{j=n+1}^{p} \Gamma\left(a_{i}+\alpha_{i} s\right) \prod_{j=m+1}^{q} \Gamma\left(1-b_{j}-\beta_{j} s\right)} z^{-s} d s,
\end{aligned}
$$

where $\mathcal{C}$ denotes the Mellin-Barness contour. Note that the FHF is recently used in wireless communication to provide closed form expressions for several system performance metrics [40]. Furthermore, it can be efficiently computed using MATHEMATICA® [41, Appendix].

Using the EGK distribution, the average symbol error probability is given in the following sections.

\section{A. Unified Average SER}

Theorem 5. The average unified SER for BPSK, PAM, approximated QAM, and approximated MPSK in $3 N T$ with EGK fading on the downlink, Laplacian inter-user interference, and maximum likelihood detector is given by

$$
\overline{S E R}=\sum_{k=1}^{n} \frac{2 a_{k}}{\Gamma(m) \Gamma\left(m_{s}\right)} \mathrm{H}_{1,2}^{2,1}\left[\begin{array}{l|c}
\beta \beta_{s} & (1,2) \\
\bar{\gamma} b_{k}^{2} & \left(m, \frac{1}{\xi}\right)\left(m_{s}, \frac{1}{\xi_{s}}\right)
\end{array}\right],
$$

where $a_{k}$ and $b_{k}$ are defined in Table I.

Proof: From (23), the average SER can be determined by solving the integral $\mathcal{I}\left(b_{k}\right)=\int_{0}^{\infty} p_{\gamma}(\gamma) e^{-b_{k} \sqrt{\gamma}}$, where $b_{k}$ are defined in Table I. Using (26), and the expression of the exponential in terms of the FHF [38, Eq. (2.9.4)], we obtain an integral of the product of two FHF, which is solved using the identity [38. Eq. (2.8.4)].

\section{B. Exact Average SER for Rectangular QAM}

The exact average SER of the QAM constellation is obtained by averaging (9) over the SIR distribution (26). The result is described in Theorem 6.
Theorem 6. The average SER of rectangular QAM in $3 N T$ with EGK fading on the downlink, Laplacian inter-user interference, and maximum likelihood detector is described in 28.

In $[28, H, \cdot, \cdot, \cdot, \cdot[\cdot, \cdot]$ is the bivariate Fox's $H$ function (BFHF) that is defined and studied in [34]. A pseudo MATLAB $^{\circledR}$ code for the BFHF is outlined in [42].

Proof: From (9), the average SER can be determined by solving an integral in the form $\int_{0}^{\infty} p_{\gamma}(\gamma) P_{Q A M}(e \mid \gamma) d \gamma$. The first two terms are similar to (30) and expressed in terms of the FHF. While the last term is solved using the Mellin representation of the BMGF [34].

\section{Exact Average SER for MPSK}

The average SER of MPSK modulation is characterized in the following theorem.

Theorem 7. The average SER of MPSK in $3 N T$ with EGK fading on the downlink, Laplacian inter-user interference, and maximum likelihood detector is described in 29].

Proof: Using a similar proof to Theorem [6, the average SER of MPSK modulation can be obtained by averaging 20] over the PDF of $\gamma$. The first term will give a result similar to [30, while the last term can be expressed in terms of the $B F H F$ using the Mellin representation of the BMGF.

\section{Special Cases of Fading and Simplification of $\overline{S E R}$}

In what follows we investigate several special cases for the EGK distribution where the average SER reduces to more simplified expression. Note that we only consider the special cases for the unified average SER given in Theorem 5, which is given in terms of $a_{k}$ and $b_{k}$ defined in Table I. On the other hand, the exact average SER of the rectangular QAM and MPSK cannot be further simplified because they are expressed in terms of the BFHF. Hence, only the approximations of the SER of QAM and MPSK (that are included in the unified formula in Theorem 5 ) are investigated in the following special cases.

Special Case 1 (Generalized- $\mathcal{K}$ fading) is obtained by setting $\xi=\xi_{s}=1$ in (30). Using the identity [39. Eq. (8.3.22)] that relates the FHF to the MGF for rational parameters, the average SER can be simplified to

$$
\overline{S E R}=\sum_{k=1}^{n} \frac{a_{k}}{\sqrt{\pi} \Gamma(m) \Gamma\left(m_{s}\right)} \mathrm{G}_{2,2}^{2,2}\left[\frac{4 m m_{s}}{\bar{\gamma} b_{k}^{2}} \mid \begin{array}{c}
1, \frac{1}{2} \\
m, m_{s}
\end{array}\right] .
$$

This expression is in terms of the MGF which is less complex in terms of computation than the FHF.

Special Case 2 ( $\mathcal{K}$ fading distribution) is a special case of the generalized- $\mathcal{K}$ fading distribution, that is obtained by setting $\xi_{s}=\xi=1$ and $m_{s}=1$. Hence the average SER can be simplified to

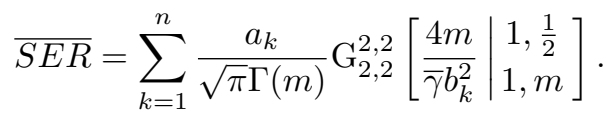




$$
\begin{aligned}
& \overline{S E R}_{Q A M}=\frac{2 \rho\left(M_{I}\right)}{\Gamma(m) \Gamma\left(m_{s}\right)} \mathrm{H}_{1,2}^{2,1}\left[\frac{\beta \beta_{s} d^{2}}{12 \bar{\gamma}} \mid \begin{array}{c}
(1,2) \\
\left(m, \frac{1}{\xi}\right)\left(m_{s}, \frac{1}{\xi_{s}}\right)
\end{array}\right]+\frac{2 \rho\left(M_{Q}\right)}{\Gamma(m) \Gamma\left(m_{s}\right)} \mathrm{H}_{1,2}^{2,1}\left[\frac{\beta \beta_{s} d^{2}}{12 \tau^{2} \bar{\gamma}} \mid \begin{array}{c}
(1,2) \\
\left(m, \frac{1}{\xi}\right)\left(m_{s}, \frac{1}{\xi_{s}}\right)
\end{array}\right]
\end{aligned}
$$

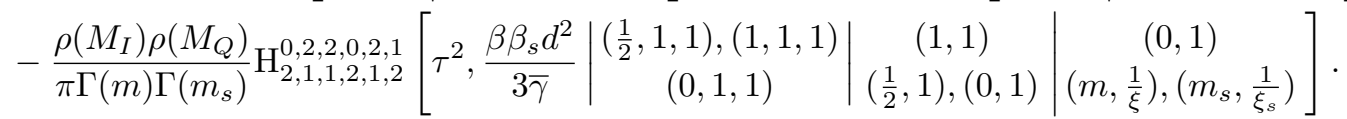

$$
\begin{aligned}
& \overline{S E R}_{M P S K}=\frac{2}{\Gamma(m) \Gamma\left(m_{s}\right)} \mathrm{H}_{1,2}^{2,1}\left[\begin{array}{l|c}
\beta \beta_{s} & (1,2) \\
4 \delta^{2} \bar{\gamma} & \left(m, \frac{1}{\xi}\right)\left(m_{s}, \frac{1}{\xi_{s}}\right)
\end{array}\right] \\
& -\frac{2 \delta}{M \sqrt{\pi}} \frac{1}{\Gamma(m) \Gamma\left(m_{s}\right)} \sum_{k=0}^{\frac{M}{4}-1} \mathrm{H}_{1,1,1,1,2,2}^{0,1,1,0,2,2}\left[\delta^{2}, \frac{4 \beta \beta_{s} \delta^{2}\left(1-\delta^{2}\right)}{\bar{\gamma}\left(\cos \left(\frac{2 k \pi}{M}\right)^{2}-\delta^{2}\right)} \mid \begin{array}{c|c|c}
\left(\frac{1}{2}, 1,1\right) & \left(\frac{1}{2}, 1\right) & (1,1),(0,1) \\
\left(-\frac{1}{2}, 1,1\right) & (0,1) & \left(m, \frac{1}{\xi}\right)\left(m_{s}, \frac{1}{\xi_{s}}\right)
\end{array}\right] .
\end{aligned}
$$

Special Case 3 (Generalized Nakagami- $m$ GNM) is obtained by setting $\xi_{s}=1$ and taking the limit $m_{s} \rightarrow+\infty$ in the EGK distribution, which makes the SER converges to

$$
\overline{S E R}=\sum_{k=1}^{n} \frac{2 a_{k} \xi}{\Gamma(m)}\left(\frac{\beta}{b_{k}^{2} \bar{\gamma}}\right)^{m \xi} \Gamma\left(2 m \xi, 0,\left(\frac{\beta}{b_{k}^{2} \bar{\gamma}}\right)^{\xi},-2 \xi\right) \text {. }
$$

Such expression can be deduced from the inverse Mellin transform of the extended incomplete Gamma function [37. Eq. (6.29)].

Special Case 4 (Nakagami- $m$ ) appears as a special case of the GNM when $\xi=1$. Using the identity [37, Eq. (6.44)] that reduces $\Gamma(\cdot, \cdot, \cdot, \cdot)$ to the Tricomi confluent hypergeometric function $U(\cdot, \cdot, \cdot)$ [27, Chapter 13], the average SER further simplifies to

$$
\overline{S E R}=\sum_{k=1}^{n} \frac{2 a_{k} \Gamma(2 m)}{4^{m} \Gamma(m)} U\left(m, \frac{1}{2}, \frac{b_{k}^{2} \bar{\gamma}}{4}\right) .
$$

Special Case 5 (Rayleigh) is the last and simplest special case, which is a special case of the Nakagami- $m$ fading for $m=1$. In this case, the SER is obtained in its simplest form as

$$
\overline{S E R}=\sum_{k=1}^{n} a_{k}\left(1-b_{k} \sqrt{\pi \bar{\gamma}} e^{b_{k}^{2} \bar{\gamma}} 4\left(b_{k} \sqrt{\frac{\bar{\gamma}}{2}}\right)\right),
$$

where $Q(\cdot)$ is the Gaussian $\mathrm{Q}$ function [20 Eq. (A.1)].

Although the average SER over any special case of fading of the EGK can be deduced from (30) by setting the quadruplet $\left(m, m_{s}, \xi, \xi_{s}\right)$ to the desired values, the previous studied special cases are highlighted because they are the most commonly used distribution to characterize the flat fading channels. Moreover, the results obtained in this section are given in their simplest forms, which require several manipulations after substituting $\left(m, m_{s}, \xi, \xi_{s}\right)$ by their desired values.

\section{NUMERICAL RESULTS}

This section shows some selected numerical results supported by Monte Carlo MATLAB ${ }^{\circledR}$ simulations to validate the developed mathematical framework and obtain insights for NLOS user pairing in 3NT FD operation. Particularly, we validate the Gaussian signaling assumption for the interfering symbol in (1), the dominance of the inter-user interference when compared to the noise in (2), and the MQAM and MPSK error rates approximations in Proposition 1 and Proposition 2, respectively.

All results are plotted against the average $\operatorname{SIR} \bar{\gamma}=\frac{P_{d} \mathcal{A}_{d}}{P_{u} \mathcal{A}_{u}}$, which is varied from $0 \mathrm{~dB}$ to $50 \mathrm{~dB}$ to provide insights for modulation scheme selection based on the average SIR values of the paired devices. When multiple devices are present, the BS should pair all NLOS devices such that the lowest SIR among the paired devices is achieved. Then, using the presented results, the BS can assign the appropriate modulation scheme for each of the paired devices based on the estimated average SIR on the interference link.

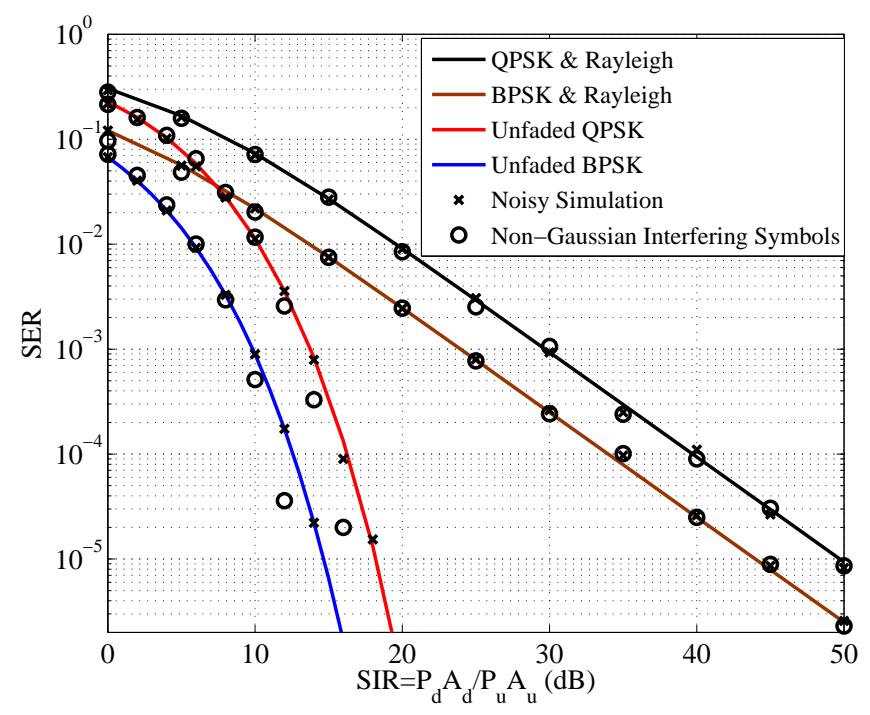

Fig. 3: SER of BPSK and QPSK schemes with and without Rayleigh fading.

Fig. 3 shows the SER of BPSK and 4-QAM (or equivalently QPSK). In Fig. 3 the analysis is validated via two simulation scenarios, namely the "Noisy Simulation" and "Non-Gaussian Interfering Symbols". In the Noisy Simulation, the received signal is perturbed by a Gaussian noise in addition to the Laplacian interference, which perfectly matches the analysis and validates the derived SER expressions. The interfering UE in Non-Gaussian Interfering Symbols scenario randomly 
and uniformly alternates between BPSK and M-QAM for $M \in\{4,16,64\}$. Hence, "Non-Gaussian Interfering Symbols" scenario validates the Gaussian signaling assumption of the interfering UEs. The figure shows that the accuracy of the Gaussian signaling assumption is better for faded downlink scenarios. This is because fading on the downlink increases the level of uncertainties in the detection process at the test UE, which puts less significance on the interfering symbol distribution from the interfering UE and vice versa. Nevertheless, the Gaussian signaling approximation always shows good accuracy in the low SIR regime, which is a critical region of operation to avoid throughput degradation.

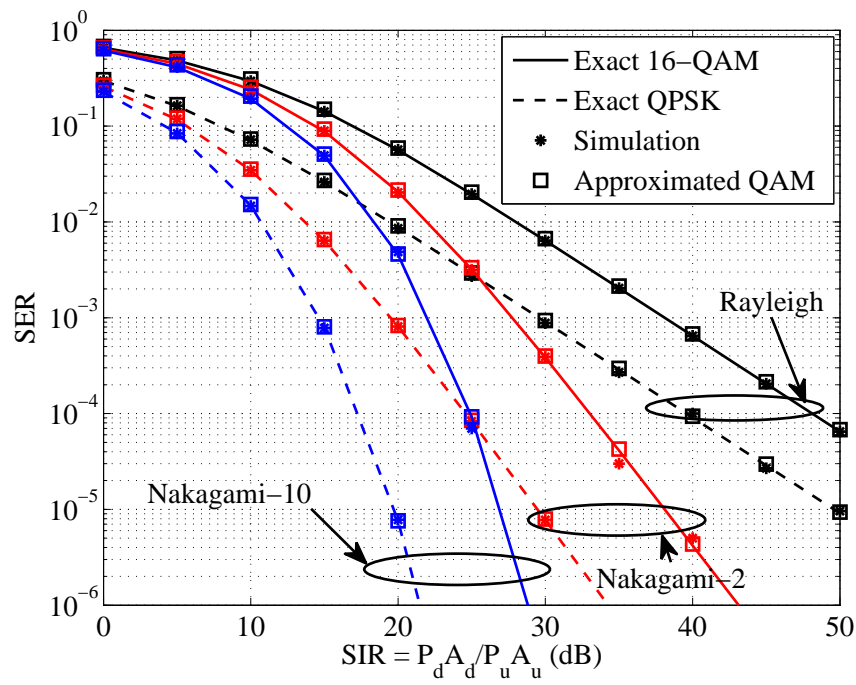

Fig. 4: SER of QPSK and 16-QAM schemes over Nakagami$m$ fading for $m=1,2$, and 10 and negligible noise. The stars denote the simulation results and the squares denote the approximation SER.

Fig. 3 and Fig. 4 show the cost of inter-user interference in terms of error probability for different modulation schemes and fading model on the downlink. Generally, the figures show that inter-user interference severely degrade the downlink performance at low SIR and that the effect of inter-user interference diminishes for high $\bar{\gamma}$ values specially for good downlink channel condition. Accordingly, based on the results of Fig. 3 and Fig. 4 along with the downlink quality of service requirement, the BS can decide the pairing SIR threshold for NLOS users. Fig. 4 also shows that the gap between the constellations decreases by increasing the fading severity (from $5 \mathrm{~dB}$ to $2 \mathrm{~dB}$ for $\mathrm{SER}=10^{-5}$ ), which is due to the dominance of the fading conditions on the error probability.

Fig. 5 is plotted to investigate the Laplacian interference dominance assumption in (2) and to show the model accuracy when varying the interference-to-noise-power-ratio (INR). The figure plots the SER of 16PSK scheme in two types of fading channels, namely, Nakagami-4 channel and Rayleigh channel. The SER is plotted versus the signal to interference plus noise ratio (SINR) (where $\sigma_{n}^{2}$ represents the noise power) for fixed noise-plus-interference power but different values for the INR. Particularly, three values of INR are investigated, $(i)$

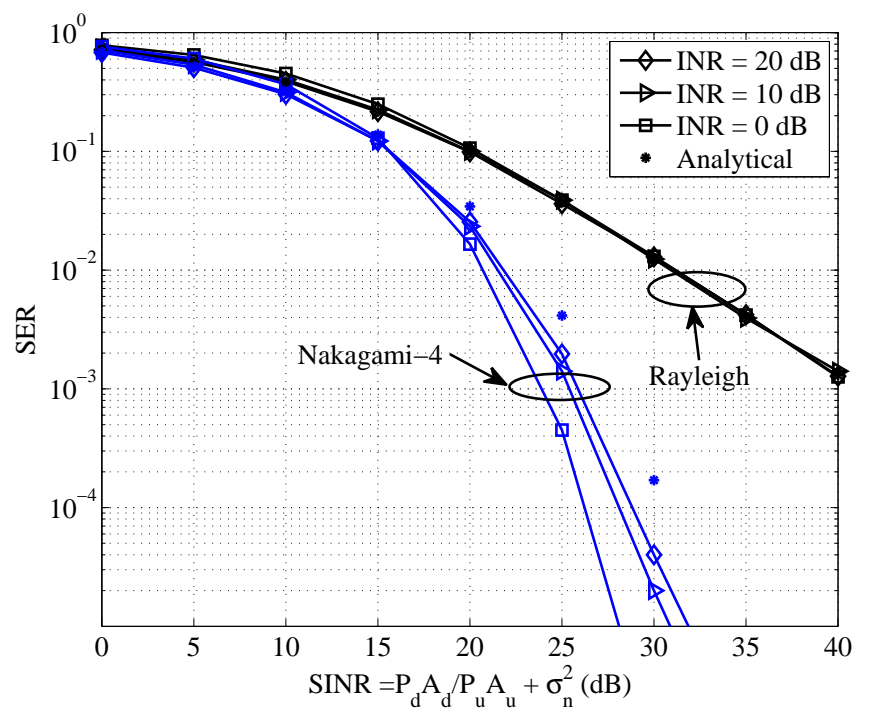

Fig. 5: SER of 16PSK scheme through Rayleigh fading and Nakagami-4 channels with different values of INR.

interference limited INR $=20 d B$, (ii) less interference limited INR $=10 d B$, and (iii) equal ratio between interference and thermal noise INR $=0 d B$. At first, the figure shows a close match between the theoretical results and simulations in all fading scenarios and INR values at low SINR regime. However, at high SINR regime, the model accuracy depends on the fading scheme. In the Rayleigh fading scenario, the noise effect is negligible and the theoretical results exactly match all INR cases in the high SINR regime. However, in Nakagami4 scenario (i.e., less fading severity) the theoretical results represent an upper-bound for the SER at high SINR regime due to the heavy tailed distribution of the Laplacian perturbation. Note that the gap between the theoretical results and simulations diminishes at sever fading (i.e., Rayleigh) and/or low SINR regime because the effect of the noise/interference tail distributions on the SER performance is not significant. That is, the error may occur at low values of interference plus noise, and hence, the tail distribution does not have a prominent effect. On the other hand, at high SINR regime and/or less sever fading, the error occurs at high interference plus noise power. Hence, the tail distribution effect becomes prominent and the gap is more noticeable.

The error rate analysis shows only the negative effect of the NLOS user paring in 3NT FD cellular networks. This is because the error rate for a given modulation scheme is a function of the SIR only which is degraded by the inter-user interference. In order to see the overall effect of the proposed NLOS user pairing criterion, we look at the throughput which is a function of both the degraded SIR as well as the improved BW. The throughput is defined as

$$
\mathcal{T}=\frac{\log _{2}(M)(1-\overline{S E R})}{t_{s}},
$$

where $\log _{2}(M)$ is the number of bits per symbol and $t_{s}$ is the symbol duration. For the HD case, we assume error free 


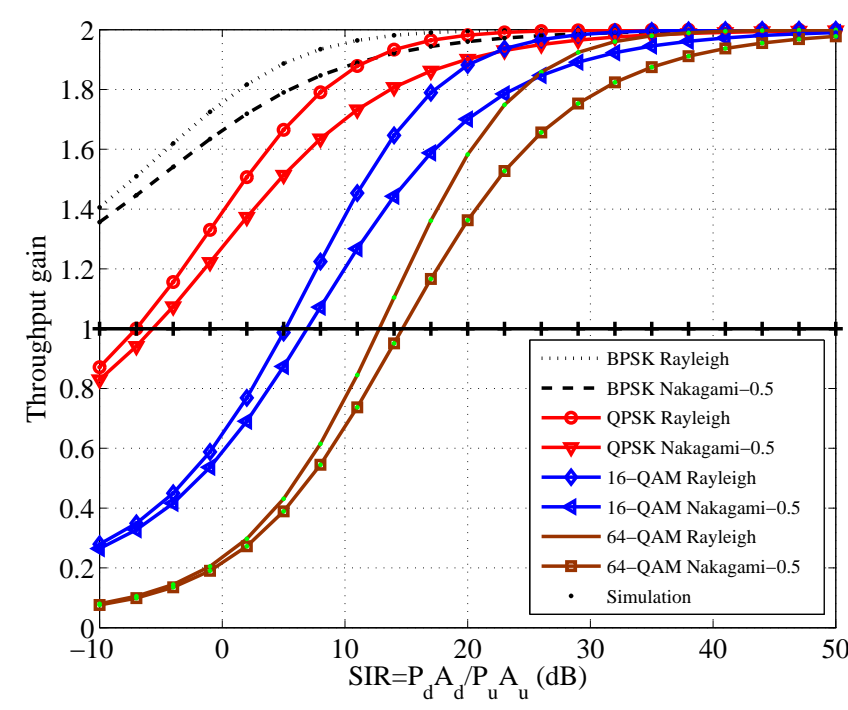

Fig. 6: Throughput Gain of BPSK and QPSK over Rayleigh and Nakagami-0.5.

transmission and channel BW of $B$. Consequently, the HD throughput is given by

$$
\mathcal{T}_{H D}=B \log _{2}(M) .
$$

For the FD case, the $3 \mathrm{NT}$ increases the channel BW to $2 B$ on the expense of inter-user interference that imposes detection errors. Consequently, the FD throughput is given by

$$
\mathcal{T}_{F D}=2 B \log _{2}(M)(1-\overline{S E R}) .
$$

For each modulation scheme, we define the throughput gain imposed by the NLOS user pairing via 3NT FD communication as

$$
\mathcal{G}=\frac{\mathcal{T}_{F D}}{\mathcal{T}_{H D}}=2(1-\overline{S E R}) .
$$

Fig. 6 shows the complete picture of the inter-user interference effect imposed by the 3NT FD by looking at the throughput gain with respect to the interference free HD scenario. Interestingly, this figure shows that the throughput gain is always greater than unity for BPSK regardless of the SIR severity. This is because the FD doubles the transmission rate while the worst case error probability for BPSK is one half. However, for higher modulation schemes, which are typical for downlink transmission, the inter-user interference can significantly degrade the effective throughout. For instance, an $80 \%$ throughout degradation occurs for 64-QAM modulation at $0 \mathrm{~dB}$ SIR. On the other hand, at high SIR regime, the proposed NLOS user pairing via 3NT doubles the effective throughput. The throughput gain, which relay on the developed error rate model, can be used to maximize the transmission rate via adaptive modulation based on the experienced SIR and fading severity. The results in Fig. 6 can be used by the BS to assign the appropriate downlink modulation scheme for each paired HD device based on the average SIR.

\section{CONCLUSION}

This paper develops a mathematical framework to study downlink error rate and throughput for half-duplex users when served by FD BSs via the 3 NT. The developed model is used to motivate long-term user pairing for user with NLOS interfering links. A comprehensive performance assessment is presented for such pairing scheme and insights into the downlink modulation scheme selection are provided. Particularly, approximating the interfering user symbols by Gaussian signals, the baseband NLOS inter-user interference is shown to be Laplacian with dependent real and imaginary parts. The paper then derives closed form expressions for the average symbol error rate for BPSK, PAM, QAM, and MPSK modulations schemes for an unfaded and then an EGK faded downlink channel. To this end, we derive the effective throughput gain imposed by NLOS user pairing via $3 \mathrm{NT}$ FD communication when compared to an idealized interference and noise free HD scenario. The results can be used to determine SIR pairing threshold based on the downlink quality of service requirements as well as to adaptively change the modulation scheme according the SIR and downlink channel condition to maximize the downlink throughput. All mathematical findings of the paper are verified via independent Monte Carlo simulations.

\section{APPENDIX A \\ PROOF OF LEMMA 1}

From the definition of the CHF, we can write

$$
\varphi_{Z}\left(t_{1}, t_{2}\right)=\mathbb{E}\left[e^{j\left(t_{1} \Re(Z)+t_{2} \Im(Z)\right)}\right] .
$$

Replacing the real and imaginary parts of $Z$ as follows in A.1, we have

$$
\begin{aligned}
\mathfrak{R}(Z) & =g_{r} x_{r}-g_{i} x_{i} \\
\mathfrak{I}(Z) & =g_{r} x_{i}+g_{i} x_{r},
\end{aligned}
$$

to obtain the following new expression of the $\mathrm{CHF}$

$$
\begin{aligned}
\varphi_{Z}\left(t_{1}, t_{2}\right)=\mathbb{E}[ & \exp \left(j x_{r}\left(t_{1} g_{r}+t_{2} g_{i}\right)\right) \\
& \left.\times \exp \left(j x_{i}\left(t_{2} g_{r}-t_{1} g_{i}\right)\right)\right] .
\end{aligned}
$$

By conditioning with respect to $g_{r}$ and $g_{i}$ (which are independent Gaussian random variables), the CHF of $Z$ can be written as the product of the CHF of $x_{r}$ and $x_{i}$, since $x_{r}$ and $x_{i}$ are independent among themselves and independent to $g_{r}$ and $g_{i}$. Now the CHF of $Z$ can be expressed as the mean over $g_{r}$ and $g_{i}$ of the conditional CHF

$$
\begin{aligned}
\varphi_{Z}\left(t_{1}, t_{2}\right)=\mathbb{E}_{g_{i}, g_{r}}[ & \varphi_{x_{r} \mid g_{i}, g_{r}}\left(t_{1} g_{r}+t_{2} g_{i}\right) \\
& \left.\times \varphi_{x_{i} \mid g_{i}, g_{r}}\left(t_{2} g_{r}-t_{1} g_{i}\right)\right] .
\end{aligned}
$$

Note that if $X$ follows a Gaussian distribution with zero mean and variance $\sigma^{2}$, its CHF is expressed as $\varphi_{X}(x)=e^{-\frac{1}{2} \sigma^{2} x^{2}}$. Using this fact in A.4 will give us the expression of $\varphi_{Z}(\cdot, \cdot)$ as

$$
\begin{aligned}
\varphi_{Z}\left(t_{1}, t_{2}\right)= & \int_{\mathbb{R}} \int_{\mathbb{R}} f_{g_{r}}\left(g_{r}\right) f_{g_{i}}\left(g_{i}\right) \\
& \times e^{-\frac{1}{4}\left(t_{1} g_{r}+t_{2} g_{i}\right)^{2}} e^{-\frac{1}{4}\left(t_{2} g_{r}-t_{1} g_{i}\right)^{2}} d g_{r} d g_{i} \\
= & \left(\frac{1}{\sqrt{\pi}} \int_{\mathbb{R}} e^{-\frac{1}{4} g^{2}\left(4+t_{1}^{2}+t_{2}^{2}\right)} d g\right)^{2} .
\end{aligned}
$$


Finally, by solving the remaining integral, the CHF gets its final expression as

$$
\varphi_{Z}\left(t_{1}, t_{2}\right)=\frac{4}{4+t_{1}^{2}+t_{2}^{2}}=\frac{1}{1+\frac{|\mathbf{t}|^{2}}{4}},
$$

where the vector $\mathbf{t}=\left(t_{1}, t_{2}\right)$. We can conclude at this point that the expression of the CHF of $Z$ is the same as the CHF of a bivariate Laplace distribution [23].

\section{APPENDIX B}

\section{PROOF OF THEOREM 1}

The CDF is defined as the primitive of the inverse Fourier transform of the CHF (primitive of the PDF that vanishes at $(-\infty,-\infty))$, defined also as

$$
\begin{aligned}
& F_{Z}\left(z_{r}, z_{i}\right)=F_{Z_{r}}\left(z_{r}\right)+F_{Z_{i}}\left(z_{i}\right)+c \\
& \quad+\frac{1}{4 \pi^{2}} \iint_{z_{r}, z_{i}} \iint_{t_{1}, t_{2}} e^{j\left(z_{r} t_{1}+z_{i} t_{2}\right)} \varphi_{Z}\left(t_{1}, t_{2}\right) d t_{1} d t_{2} d z_{r} d z_{i},
\end{aligned}
$$

where $F_{Z_{r}}(\cdot)$ and $F_{Z_{i}}(\cdot)$ are the CDFs of $Z_{r}$ and $Z_{i}$ respectively, and $c$ is a real constant to be determined. In the following analysis we are focusing on the last part (four integrals), namely $\bar{F}_{Z}\left(z_{r}, z_{i}\right)$, which is the cumulative CDF (CCDF).

Looking at the CHF, one notice that it is an even function on both variables, so the Fourier transform becomes a cosine transform

$$
\begin{aligned}
\bar{F}_{Z}\left(z_{r}, z_{i}\right)= & \frac{1}{\pi^{2}} \iint_{z_{r}, z_{i}} \int_{0}^{\infty} \int_{0}^{\infty} \cos \left(z_{r} t_{1}\right) \cos \left(z_{i} t_{2}\right) \\
& \times \varphi_{Z}\left(t_{1}, t_{2}\right) d t_{1} d t_{2} d z_{r} d z_{i} \\
= & \frac{4}{\pi^{2}} \int_{0}^{\infty} \int_{0}^{\infty} \frac{\sin \left(z_{r} t_{1}\right) \sin \left(z_{i} t_{2}\right)}{t_{1} t_{2}\left(4+t_{1}^{2}+t_{2}^{2}\right)} d t_{1} d t_{2} .
\end{aligned}
$$

Using the representation of the sine function in terms of the Meijer's G function (MGF) [38, Eq. (2.9.7)] and its MellinBarnes representation [38, Eq. (1.1.2)], yields

$$
\begin{aligned}
\sin (x) & =\sqrt{\pi} \mathrm{G}_{0,2}^{1,0}\left[\frac{x^{2}}{4} \mid \overline{\frac{1}{2}, 0}\right] \\
& =\sqrt{\pi} \frac{1}{2 \pi j} \int_{\mathcal{L}} \frac{\Gamma\left(\frac{1}{2}+s\right)}{\Gamma(1-s)}\left(\frac{x}{2}\right)^{-2 s} d s .
\end{aligned}
$$

Substituting the expressions of the sine function (B.2) in B.1], we get another expression of the $\mathrm{CCDF}$ as

$$
\begin{gathered}
\bar{F}_{Z}\left(z_{r}, z_{i}\right)=\frac{4}{\pi} \frac{1}{(2 \pi j)^{2}} \int_{\mathcal{L}_{1}} \int_{\mathcal{L}_{2}} \frac{\Gamma\left(\frac{1}{2}+s\right) \Gamma\left(\frac{1}{2}+t\right)}{\Gamma(1-s) \Gamma(1-t)} \\
\left(\frac{z_{r}}{2}\right)^{-2 s}\left(\frac{z_{i}}{2}\right)^{-2 t} \int_{0}^{\infty} \int_{0}^{\infty} \frac{t_{1}^{-2 s-1} t_{2}^{-2 t-1}}{4+t_{1}^{2}+t_{2}^{2}} d t_{1} d t_{2} d s d t .
\end{gathered}
$$

The last double integral has a closed from solution which is equal to

$$
\begin{aligned}
\int_{0}^{\infty} \int_{0}^{\infty} \frac{t_{1}^{-2 s-1} t_{2}^{-2 t-1}}{4+t_{1}^{2}+t_{2}^{2}} d t_{1} d t_{2} & =2^{-4-2 s-2 t} \Gamma(1+s+t) \\
\times & \frac{\Gamma(1-s) \Gamma(1-t) \Gamma(s) \Gamma(t)}{\Gamma(1+s) \Gamma(1+t)} .
\end{aligned}
$$

This result is valid for $s \in \mathcal{L}_{1}$ and $t \in \mathcal{L}_{2}$. Hence (B.3) can be re-written as

$$
\begin{aligned}
\bar{F}_{Z}\left(z_{r}, z_{i}\right)= & \frac{1}{4 \pi} \frac{1}{(2 \pi j)^{2}} \int_{\mathcal{L}_{1}} \int_{\mathcal{L}_{2}} \Gamma(1+s+t) \Gamma(s) \Gamma(t) \\
& \times \frac{\Gamma\left(\frac{1}{2}+s\right) \Gamma\left(\frac{1}{2}+t\right)}{\Gamma(1+s) \Gamma(1+t)} z_{r}^{-2 s} z_{i}^{-2 t} d s d t \\
= & \frac{1}{4 \pi} \mathrm{G}_{1,0,2,1,2,1}^{0,1,0,2,0,2}\left[\frac{1}{z_{r}^{2}}, \frac{1}{z_{i}^{2}}|\longrightarrow| \begin{array}{c|c|c}
\frac{1}{2}, 1 & \frac{1}{2}, 1 \\
0 & 0
\end{array}\right] .
\end{aligned}
$$

Since $Z_{r}$ and $Z_{i}$ have marginal Laplace distribution, their $\mathrm{CDFs}$ have the same form that is given by

$$
\left\{\begin{array}{l}
F_{Z_{r}}\left(z_{r}\right)=1-\frac{1}{2} e^{-2 z_{r}} \\
F_{Z_{i}}\left(z_{i}\right)=1-\frac{1}{2} e^{-2 z_{i}} .
\end{array}\right.
$$

Finally it is easy to proof that $c=-1$, and thus end of the proof.

\section{APPENDIX C \\ ProOF OF COROLlary 1.1}

The PDF is the derivative of the CDF with respect to $z_{r}$ and $z_{i}, f_{Z}\left(z_{r}, z_{i}\right)=\frac{\partial^{2} F_{Z}\left(z_{r}, z_{i}\right)}{\partial z_{r} \partial z_{i}}$. While both marginal CDF components vanish after the second derivation, only the CCDF component is remaining. Therefore, from $B .5$ and with the help of a change of variable, the PDF of $Z$ can be written as

$$
\begin{aligned}
f_{Z}\left(z_{r}, z_{i}\right)= & \frac{1}{\pi} \frac{1}{(2 \pi j)^{2}} \int_{\mathcal{L}_{1}} \int_{\mathcal{L}_{2}} \Gamma(1+s+t) \\
= & \frac{1}{\pi} \frac{1}{(2 \pi j)^{2}} \int_{\mathcal{L}_{1}} \Gamma(s) z_{i}^{-2 s} \\
& \int_{\mathcal{L}_{2}} \Gamma(s-t) \Gamma(t)\left(\frac{z_{r}}{z_{i}}\right)^{-2 t} d t d s .
\end{aligned}
$$

Using the identity [38, Eq. (2.9.5)] to solve the second integral, we can write

$$
\begin{aligned}
\frac{1}{2 \pi j} \int_{\mathcal{L}_{2}} \Gamma(s-t) \Gamma(t)\left(\frac{z_{r}}{z_{i}}\right)^{-2 t} d t & =\mathrm{G}_{1,1}^{1,1}\left[\frac{z_{r}^{2}}{z_{i}^{2}} \mid \begin{array}{c}
1-s \\
0
\end{array}\right] \\
& =\Gamma(s)\left(1+\frac{z_{r}^{2}}{z_{i}^{2}}\right)^{-s} .
\end{aligned}
$$

Finally, the PDF is simplified to the desired final results

$$
\begin{aligned}
f_{Z}\left(z_{r}, z_{i}\right) & =\frac{1}{\pi} \frac{1}{2 \pi j} \int_{\mathcal{L}_{1}} \Gamma(s) \Gamma(s)\left(z_{r}^{2}+z_{i}^{2}\right)^{-s} d s \\
& =\frac{1}{\pi} \mathrm{G}_{0,2}^{2,0}\left[z_{r}^{2}+z_{i}^{2} \mid \bar{\square}\right] \\
& =\frac{2}{\pi} K_{0}\left(2 \sqrt{z_{r}^{2}+z_{i}^{2}}\right) .
\end{aligned}
$$

The last equality is obtained using the identities [38, Eq. (2.9.31) \& Eq. (2.9.39)]. 


\section{APPENDIX D}

\section{PROOF OF THEOREM 3}

A rectangular QAM constellation is formed by $M=$ $M_{I} M_{Q}$ symbols. These symbols can be classified as follows: 4 corner symbols, $2\left(M_{I}-2\right)$ edge symbols with decision distance $d_{I}, 2\left(M_{Q}-2\right)$ edge symbols with decision distance $d_{Q}$, and $\left(M_{I}-2\right)\left(M_{Q}-2\right)$ inner symbols. To compute the conditional error probability, we need to get the probability of error of each symbol and then the SER is obtained by averaging these probabilities over the probability of occurrence of each symbol. Let us begin with the corner symbols. Actually the probability of error of the corner symbol can be given as

$$
\begin{aligned}
P_{e}^{c o r} & =1-\int_{-\frac{d_{I}}{2}}^{\infty} \int_{-\frac{d_{Q}}{2}}^{\infty} f_{Z}(x, y) d x d y=1-F_{Z}\left(\frac{d_{I}}{2}, \frac{d_{Q}}{2}\right) \\
& =\frac{1}{2}\left(e^{-d_{I}}+e^{-d_{Q}}\right)-\bar{F}_{Z}\left(\frac{d_{I}}{2}, \frac{d_{Q}}{2}\right),
\end{aligned}
$$

where $\bar{F}_{Z}(\cdot, \cdot)$ is defined in $(\mathrm{B} .5)$.

The calculation of the probability of error of the edge points is similar for the in-phase point and the quadraturephase points, hence we are focusing on the in-phase point. Furthermore, the probability of error detection of an in-phase edge symbol is obtained as

$$
\begin{aligned}
P_{e}^{e d g, I} & =1-\int_{-\frac{d_{I}}{2}}^{\infty} \int_{-\frac{d_{Q}}{2}}^{\frac{d_{Q}}{2}} f_{Z}(x, y) d x d y \\
& =2-\frac{1}{2} e^{-d_{I}}-2 F_{Z}\left(\frac{d_{I}}{2}, \frac{d_{Q}}{2}\right) \\
& =\frac{1}{2}\left(e^{-d_{I}}+2 e^{-d_{Q}}\right)-2 \bar{F}_{Z}\left(\frac{d_{I}}{2}, \frac{d_{Q}}{2}\right) .
\end{aligned}
$$

Per consequence, the probability of error detection of a quadrature phase edge symbol is given by

$$
\begin{aligned}
P_{e}^{e d g, Q} & =\frac{1}{2}\left(e^{-d_{Q}}+2 e^{-d_{I}}\right)-2 \bar{F}_{Z}\left(\frac{d_{Q}}{2}, \frac{d_{I}}{2}\right) \\
& =\frac{1}{2}\left(e^{-d_{Q}}+2 e^{-d_{I}}\right)-2 \bar{F}_{Z}\left(\frac{d_{I}}{2}, \frac{d_{Q}}{2}\right) .
\end{aligned}
$$

Finally, the probability of error detection of an inner symbol is obtained as

$$
\begin{aligned}
P_{e}^{i n n} & =1-\int_{-\frac{d_{I}}{2}}^{\frac{d_{I}}{2}} \int_{-\frac{d_{Q}}{2}}^{\frac{d_{Q}}{2}} f_{Z}(x, y) d x d y \\
& =e^{-d_{I}}+e^{-d_{Q}}-4 \bar{F}_{Z}\left(\frac{d_{I}}{2}, \frac{d_{Q}}{2}\right) .
\end{aligned}
$$

Hence, assuming equiprobable symbols, the SER of rectangular QAM is derived as

$$
\begin{aligned}
& P_{Q A M}(e \mid \gamma)=\frac{1}{M_{I} M_{Q}}\left(4 P_{e}^{c o r}+2\left(M_{Q}-2\right) P_{e}^{e d g, Q}\right. \\
& \left.\quad+2\left(M_{I}-2\right) P_{e}^{e d g, I}+\left(M_{Q}-2\right)\left(M_{I}-2\right) P_{e}^{i n n}\right)
\end{aligned}
$$

Now by replacing the decision distances by their values $d_{I}=\frac{2 \sqrt{3 \gamma}}{d}, d_{Q}=\tau d_{I}$, using the parameter $d$ from (9), and after some algebraic manipulation, the result in Theorem 3 is obtained easily.

\section{APPENDIX E \\ PROOF OF THEOREM 4}

First, the probability that $Y_{1}>0$ is equivalent to $\operatorname{Pr}[\tan (\varphi-$ $\theta) I-Q \leq 0]$, because $\varphi \in\left[0, \frac{\pi}{4}-\frac{2 \pi}{M}\right]$ and $\theta=\frac{\pi}{M}$. Now let's call $X=\tan (\varphi-\theta) I-Q$, then the PDF of $X$ can be written as

$$
f_{X}(x)=\int_{\mathbb{R}} f_{I, Q}(u, \tan (\varphi-\theta) u-x) d u .
$$

Which gives the following expression of the first term of 17 as

$$
\begin{aligned}
\operatorname{Pr}\left[Y_{1} \geq 0\right] & =\operatorname{Pr}[X \leq 0]=\operatorname{Pr}[-X \geq 0] \\
& =\int_{0}^{\infty} \int_{\mathbb{R}} f_{I, Q}(u, \tan (\varphi-\theta) u+x) d u d x \\
& =\frac{2 \gamma}{\pi} \int_{0}^{\infty} \int_{\mathbb{R}} K_{0}(2 \sqrt{\gamma A(u, x)}) d u d x,
\end{aligned}
$$

where $A(u, x)=(u+\cos \varphi)^{2}+(\tan (\varphi-\theta) u+x+\sin \varphi)^{2}$. Using a change of variable and the fact that the marginal of (6) is Laplacian (i.e. $\int_{\mathbb{R}} \frac{2}{\pi} K_{0}\left(2 \sqrt{u^{2}+v^{2}}\right) d u=e^{-2|v|}$ ), E.2. can be further simplified to

$$
\operatorname{Pr}\left[Y_{1} \geq 0\right]=\frac{1}{2} e^{-2 \sin \theta \sqrt{\gamma}} .
$$

The same analysis can be used to compute the second term of (17), and a similar result can be easily obtained

$$
\operatorname{Pr}\left[Y_{2} \leq 0\right]=\frac{1}{2} e^{-2 \sin \theta \sqrt{\gamma}} .
$$

Finally, to compute the last term in (17) (i.e. $\operatorname{Pr}\left[Y_{1} \geq 0, Y_{2} \leq\right.$ $0]$ ), we define $W_{1}=Q-\tan (\varphi-\theta) I$ and $W_{2}=\tan (\varphi+\theta) I-$ $Q$, then we have $\operatorname{Pr}\left[Y_{1} \geq 0, Y_{2} \leq 0\right]=\operatorname{Pr}\left[W_{1} \geq 0, W_{2} \leq 0\right]$. The PDF of the couple $\left(W_{1}, W_{2}\right)$ can be written, using the PDF of $(I, Q)$, as

$$
\begin{aligned}
& f_{W_{1}, W_{2}}\left(w_{1}, w_{2}\right)=\frac{2 \gamma}{\pi} \frac{\cos \varphi^{2}-\sin \theta^{2}}{\sin 2 \theta} \\
& K_{0}\left(2 \sqrt{\gamma} \frac{\cos \varphi^{2}-\sin \theta^{2}}{\sin 2 \theta} \sqrt{A_{1}\left(w_{1}, w_{2}\right)}\right),
\end{aligned}
$$

where $A_{1}\left(w_{1}, w_{2}\right)=\left(w_{1}+w_{2}+\cos \varphi\right)^{2}+\left(\tan (\varphi+\theta) w_{1}+\right.$ $\left.\tan (\varphi-\theta) w_{2}+\sin \varphi\right)^{2}$. The desired probability of error can be written as

$$
\begin{aligned}
\operatorname{Pr}\left[Y_{1} \geq 0, Y_{2} \leq 0\right] & =\operatorname{Pr}\left[W_{1} \geq 0, W_{2} \leq 0\right] \\
& =\int_{0}^{\infty} \int_{0}^{\infty} f_{W_{1}, W_{2}}\left(w_{1}, w_{2}\right) d w_{1} d w_{2} .
\end{aligned}
$$

By developing the squares in $A_{1}\left(w_{1}, w_{2}\right)$ and using a change of variable, E.6) can be expressed as

$$
\begin{aligned}
& \operatorname{Pr}\left[Y_{1} \geq 0, Y_{2} \leq 0\right]=\frac{1}{2 \pi \sin 2 \theta} \\
& \times \int_{B}^{\infty} \int_{B}^{\infty} K_{0}\left(\frac{1}{\sin 2 \theta} \sqrt{u^{2}+v^{2}+2 u v \cos 2 \theta}\right) d u d v,
\end{aligned}
$$

where $B=\sqrt{\gamma} \frac{\cos \varphi^{2}-\sin \theta^{2}}{\cos \theta}$.

An integral representation of the Bessel function, available in [43. Eq. (2.13)], $K_{0}(x)=\frac{1}{2} \int_{0}^{\infty} \frac{1}{t} e^{-t-\frac{x^{2}}{4 t}}$, can be used in E.7). Hence we obtain a double incomplete integral of an exponential with square argument, such term can be solved 
using the two dimensional Gaussian $Q$ function $Q(\cdot, \cdot, \cdot)[44$ Eq. (4.3)]. Consequently, E.7) can be reduced to one integral as follows

$$
\operatorname{Pr}\left[Y_{1} \geq 0, Y_{2} \leq 0\right]=\int_{0}^{\infty} e^{-t} Q\left(\frac{B}{\sqrt{2 t}}, \frac{B}{\sqrt{2 t}},-\cos 2 \theta\right) d t
$$

On the other hand, from [44, Eq. (4.7)], the two dimensional $Q$ function can be reduced to only one integral if its first two arguments are equal, $Q(x, x, \alpha)=$ $\frac{1}{\pi} \int_{0}^{\frac{\pi}{4}} \frac{\sqrt{1-\alpha^{2}}}{1-\alpha \sin 2 \Phi} e^{-\frac{x^{2}}{2} \frac{1-\alpha \sin 2 \Phi}{\left(1-\alpha^{2}\right) \sin ^{2} \Phi}} d \Phi$, which is the case in E.8. Using a suitable change of variable, and the integral representation of $K_{1}(\cdot)$, E.8 can be re-written as

$$
\operatorname{Pr}\left[Y_{1} \geq 0, Y_{2} \leq 0\right]=\frac{B}{2 \pi} \int_{1}^{\infty} \frac{1}{\sqrt{u^{2}-u \sin ^{2} \theta}} K_{1}\left(\frac{B \sqrt{u}}{\sin \theta}\right) d u .
$$

An alternative expression of the Bessel function and the first term that contains the square root in terms of the Meijer's G function (MGF) is available in [38, Eq. (2.9.19)] and [38, Eq. (2.9.6)], respectively

$$
\begin{gathered}
K_{1}(x)=\frac{1}{x} \mathrm{G}_{0,2}^{2,0}\left[\frac{x^{2}}{4} \mid \begin{array}{c}
- \\
0,1
\end{array}\right] \\
\frac{1}{\sqrt{1-x}}=\sqrt{\pi} \mathrm{G}_{1,1}^{1,0}\left[\begin{array}{c|c}
1 / 2 \\
0
\end{array}\right] .
\end{gathered}
$$

These alternative expressions transform the integral in E.9) as an incomplete integral of the product of two MGFs, which can be transformed also to an integral of 3 MGFs

$$
\begin{aligned}
& \operatorname{Pr}\left[Y_{1} \geq 0, Y_{2} \leq 0\right]=\frac{\sin \theta}{2 \sqrt{\pi}} \int_{0}^{\infty} u^{-3 / 2} \mathrm{G}_{1,1}^{0,1}\left[x \mid \begin{array}{l}
1 \\
0
\end{array}\right]
\end{aligned}
$$

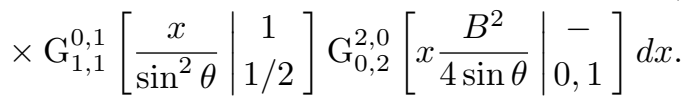

Such integral can be easily solved using [34, Eq. (2.3)] to get the results of Theorem 4 and finish the proof.

\section{APPENDIX $F$}

\section{SPeCial CASES OF THE EGK Distribution}

\section{REFERENCES}

[1] S. Hong, J. Brand, J. Choi, M. Jain, J. Mehlman, S. Katti, and P. Levis, "Applications of self-interference cancellation in 5G and beyond," IEEE Commun. Mag., vol. 52, no. 2, pp. 114-121, Feb. 2014.

[2] M. Zhou, L. Song, and Y. Li, "Joint transmit and receive antennas selection for full duplex MIMO systems," in Proc. of the IEEE Global Communications Conference (GLOBECOM'2014), Austin, TX, USA, Dec. 2014, pp. 3838-3843.

[3] N. Pappas, M. Kountouris, A. Ephremides, and A. Traganitis, "Relayassisted multiple access with full-duplex multi-packet reception," IEEE Transactions on Wireless Communications, vol. 14, no. 7, pp. 3544 3558, July 2015.

[4] A. Sabharwal, P. Schniter, D. Guo, D. Bliss, S. Rangarajan, and R. Wichman, "In-band full-duplex wireless: Challenges and opportunities," IEEE J. Select. Areas Commun, vol. 32, no. 9, pp. 1637-1652, Sep. 2014.

[5] J. I. Jain, Mayank Choi, T. Kim, D. Bharadia, S. Seth, K. Srinivasan, P. Levis, S. Katti, and P. Sinha, "Practical, real-time, full duplex

\begin{tabular}{|c|c|c|c|c|}
\hline Envelope Distribution & $m$ & $\xi$ & $m_{s}$ & $\xi_{s}$ \\
\hline \multirow{2}{*}{ Rayleigh } & 1 & 1 & $\infty$ & 1 \\
\hline & $\infty$ & 1 & 1 & 1 \\
\hline \multirow{2}{*}{ Maxwell } & $3 / 2$ & 1 & $\infty$ & 1 \\
\hline & $\infty$ & 1 & $3 / 2$ & 1 \\
\hline \multirow{2}{*}{ Half-Normal } & $1 / 2$ & 1 & $\infty$ & 1 \\
\hline & $\infty$ & 1 & $1 / 2$ & 1 \\
\hline \multirow{2}{*}{ Exponential } & 1 & $1 / 2$ & $\infty$ & 1 \\
\hline & $\infty$ & 1 & 1 & $1 / 2$ \\
\hline \multirow{2}{*}{ Weibull } & 1 & $\xi$ & $\infty$ & 1 \\
\hline & $\infty$ & 1 & 1 & $\xi_{s}$ \\
\hline \multirow{2}{*}{ Nakagami- $m$} & $m$ & 1 & $\infty$ & 1 \\
\hline & $\infty$ & 1 & $m_{s}$ & 1 \\
\hline \multirow{2}{*}{ Generalized Nakagami } & $m$ & $\xi$ & $\infty$ & 1 \\
\hline & $\infty$ & 1 & $m_{s}$ & $\xi_{s}$ \\
\hline \multirow{2}{*}{ Gamma } & $m$ & $1 / 2$ & $\infty$ & 1 \\
\hline & $\infty$ & 1 & $m_{s}$ & $1 / 2$ \\
\hline \multirow{2}{*}{ Generalized Gamma } & $m$ & $\xi / 2$ & $\infty$ & 1 \\
\hline & $\infty$ & 1 & $m_{s}$ & $\xi_{s} / 2$ \\
\hline \multirow{2}{*}{ Half-Normal-Exponential } & $1 / 2$ & 1 & 1 & 1 \\
\hline & 1 & 1 & $1 / 2$ & 1 \\
\hline \multirow{2}{*}{ Half-Normal-Gamma } & $1 / 2$ & 1 & $m_{s}$ & 1 \\
\hline & $m$ & 1 & $1 / 2$ & 1 \\
\hline \multirow{2}{*}{ GNM-Lognormal } & $\infty$ & 0 & $m_{s}$ & $\xi_{s}$ \\
\hline & $m$ & $\xi$ & $\infty$ & 0 \\
\hline \multirow{2}{*}{ Suzuki } & $\infty$ & 0 & 1 & 1 \\
\hline & 1 & 1 & $\infty$ & 0 \\
\hline Rayleigh-Exponential & 1 & 1 & 1 & 1 \\
\hline \multirow{2}{*}{ Maxwell-Lognormal } & $\infty$ & 0 & $3 / 2$ & 1 \\
\hline & $3 / 2$ & 1 & $\infty$ & 0 \\
\hline \multirow{2}{*}{ Maxwell-Exponential } & 1 & 1 & $3 / 2$ & 1 \\
\hline & $3 / 2$ & 1 & 1 & 1 \\
\hline \multirow{2}{*}{ Maxwell-Gamma } & $m$ & 1 & $3 / 2$ & 1 \\
\hline & $3 / 2$ & 1 & $m_{s}$ & 1 \\
\hline \multirow{2}{*}{ Weibull-Lognormal } & $\infty$ & 0 & 1 & $\xi_{s}$ \\
\hline & 1 & $\xi$ & $\infty$ & 0 \\
\hline \multirow{2}{*}{ Weibull-Exponential } & 1 & 1 & 1 & $\xi_{s}$ \\
\hline & 1 & $\xi$ & 1 & 1 \\
\hline Weibull-Weibull & 1 & $\xi$ & 1 & $\xi_{s}$ \\
\hline \multirow{2}{*}{ Weibull-Gamma } & $m$ & 1 & 1 & $\xi_{s}$ \\
\hline & 1 & $\xi$ & $m_{s}$ & 1 \\
\hline \multirow{2}{*}{ Nakagami-Lognormal } & $\infty$ & 0 & $m_{s}$ & 1 \\
\hline & $m$ & 1 & $\infty$ & 0 \\
\hline \multirow{2}{*}{ K-Distribution } & $m$ & 1 & 1 & 1 \\
\hline & 1 & 1 & $m_{s}$ & 1 \\
\hline Generalized-K & $m$ & 1 & $m_{s}$ & 1 \\
\hline GNM-Exponential & 1 & 1 & $m_{s}$ & $\xi_{s}$ \\
\hline Uivivi-Exponential & $m$ & $\xi$ & 1 & 1 \\
\hline GNM-Weibull & $m$ & $\xi$ & 1 & $\xi_{s}$ \\
\hline & 1 & $\xi$ & $m_{s}$ & $\xi_{s}$ \\
\hline GNM-Gamma & $m$ & 1 & $m_{s}$ & $\xi_{s}$ \\
\hline & $m$ & $\xi$ & $m_{s}$ & 1 \\
\hline
\end{tabular}
wireless," in Proc. of the 17th Annu. Int. Conf. on Mobile Computing and Networking (MobiCom'2011), Las Vegas, NV, USA, Sep. 2011, pp. 301-312.
TABLE II: Some Special Cases of the EGK Distribution

[6] J. Bai and A. Sabharwal, "Distributed full-duplex via wireless sidechannels: Bounds and protocols," IEEE Trans. on Wireless Commun., vol. 12, no. 8, pp. 4162-4173, August 2013.

[7] H. Zhao, J. Wang, and Y. Tang, "Performance analysis of RF selfinterference cancellation in broadband full duplex systems," in Proc. of the IEEE International Conference on Communications Workshops (ICC'2016), Kuala Lumpur, Malaysia, May 2016, pp. 175-179.

[8] K. Sundaresan, M. Khojastepour, E. Chai, and S. Rangarajan, "Fullduplex without strings: Enabling full-duplex with half-duplex clients," in Proc. of the 20th Annu. Int. Conf. on Mobile Computing and Networking (MobiCom'2014), Maui, Hawaii, USA, Sep. 2014.

[9] K. M. Thilina, H. Tabassum, E. Hossain, and D. I. Kim, "Medium access control design for full duplex wireless systems: challenges and approaches," IEEE Communications Magazine, vol. 53, no. 5, pp. 112120, May 2015.

[10] M. Mohammadi, H. A. Suraweera, Y. Cao, I. Krikidis, and C. Tellambura, "Full-duplex radio for uplink/downlink wireless access with spatially random nodes," IEEE Transactions on Communications, vol. 63 no. 12, pp. 5250-5266, Dec 2015.

[11] M. Feng, S. Mao, and T. Jiang, "Joint duplex mode selection, channel allocation, and power control for full-duplex cognitive femtocell networks," Dig. Commun. and Net., vol. 1, no. 1, pp. 30-44, 2015.

[12] G. C. Alexandropoulos, M. Kountouris, and I. Atzeni, "User scheduling and optimal power allocation for full-duplex cellular networks," in Proc. 
of the IEEE 17th International Workshop on Signal Processing Advances in Wireless Communications (SPAWC'2016), Edinburgh, UK, July 2016, pp. 1-6.

[13] J. M. B. da Silva, G. Fodor, and C. Fischione, "Spectral efficient and fair user pairing for full-duplex communication in cellular networks," IEEE Transactions on Wireless Communications, vol. 15, no. 11, pp. 7578-7593, Nov 2016

[14] H. Soury, H. ElSawy, and M.-S. Alouini, "Error rates of a full-duplex system over EGK fading channels subject to Laplacian interference," in Proc. of the IEEE International Conference on Communications (ICC'2017), Paris, France, Accepted, May 2017.

[15] F. Yilmaz and M.-S. Alouini, "A new simple model for composite fading channels: Second order statistics and channel capacity," in Proc. of the IEEE 7th Int. Symp. on Wireless Commun. Syst. (ISWCS'2010), York, UK, Sept. 2010, pp. 676-680.

[16] C. Psomas and I. Krikidis, "Outage analysis of full-duplex architectures in cellular networks," in Vehicular Technology Conf. (VTC Spring), 2015 IEEE 81st, May 2015, pp. 1-5.

[17] M. Mohammadi, H. A. Suraweera, I. Krikidis, and C. Tellambura, "Fullduplex radio for uplink/downlink transmission with spatial randomness," in Proc. IEEE Int. Conf. Commun. (ICC), London, UK, Jun. 2015, pp. 1908-1913.

[18] S. Goyal, P. Liu, S. Hua, and S. Panwar, "Analyzing a full-duplex cellular system," in Proc. 47th Annu. Conf. on Information Sciences and Systems (CISS), Mar. 2013, pp. 1-6.

[19] A. AlAmmouri, H. ElSawy, and M. S. Alouini, "Flexible design for $\alpha$-duplex communications in multi-tier cellular networks," IEEE Transactions on Communications, vol. 64, no. 8, pp. 3548-3562, Aug 2016.

[20] H. Soury, F. Yilmaz, and M.-S. Alouini, "Average bit error probability of binary coherent signaling over generalized fading channels subject to additive generalized Gaussian noise," IEEE Commun. Lett., vol. 16, no. 6, pp. 785-788, June 2012 .

[21] A. Giorgetti and M. Chiani, "Influence of fading on the Gaussian approximation for BPSK and QPSK with asynchronous cochannel interference," IEEE Trans. on Wireless Commun., vol. 4, no. 2, pp. 384 389, March 2005.

[22] L. H. Afify, H. ElSawy, T. Y. Al-Naffouri, and M.-S. Alouini, "The influence of Gaussian signaling approximation on error performance in cellular networks," IEEE Communications Letters, vol. 19, no. 12, pp. 2202-2205, Dec 2015.

[23] T. Eltoft, T. Kim, and T.-W. Lee, "On the multivariate Laplace distribution," IEEE Sig. Proces. Lett., vol. 13, no. 5, pp. 300-303, May 2006.

[24] I. Ansari, S. Al-Ahmadi, F. Yilmaz, M.-S. Alouini, and H. Yanikomeroglu, "A new formula for the BER of binary modulations with dual-branch selection over generalized-K composite fading channels," IEEE Trans. Commun., vol. 59, no. 10, pp. 2654-2658, Oct. 2011.

[25] R. K. Mallik, "Distribution of inner product of two complex Gaussian vectors and its application to MPSK performance," in IEEE International Conference on Communications (ICC'2008), Beijing, China, May 2008, pp. $4616-4620$.

[26] N. O'Donoughue and J. M. F. Moura, "On the product of independent complex Gaussians," IEEE Transactions on Signal Processing, vol. 60, no. 3, pp. 1050-1063, March 2012.

[27] M. Abramowitz and I. A. Stegun, Handbook of Mathematical Functions with Formulas, Graphs, and Mathematical Tables, ser. National
Bureau of Standards Applied Mathematics Series. New York: Dover Publications, 1964.

[28] H. Soury and M.-S. Alouini, "Symbol error rate of MPSK over EGK channels perturbed by a dominant additive Laplacian noise," IEEE Trans. on Commun., vol. 63, no. 7, pp. 2511-2523, Jul. 2015.

[29] S. Jiang and N. C. Beaulieu, "Precise BER computation for binary data detection in bandlimited white Laplace noise," IEEE Trans. Commun., vol. 59, no. 6, pp. 1570-1579, Jun. 2011.

[30] N. C. Beaulieu and D. J. Young, "Designing time-hopping ultrawide bandwidth receivers for multiuser interference environments," Proceedings of the IEEE, vol. 97, no. 2, pp. 255-284, Feb. 2009.

[31] S. Jiang and N. Beaulieu, "BER of antipodal signaling in Laplace noise," in Proc. of the 25th Biennial Symposium on Communications (QBSC'2010), Kingston, ON, Canada, May 2010, pp. 110-113.

[32] M. W. Thompson and H.-S. Chang, "Coherent detection in Laplace noise," IEEE Trans. Aerosp. Electron. Syst., vol. 30, no. 2, pp. 452461, Apr. 1994.

[33] B. M. Hochwald, T. L. Marzetta, and V. Tarokh, "Multiple-antenna channel hardening and its implications for rate feedback and scheduling," IEEE Transactions on Information Theory, vol. 50, no. 9, pp. 18931909, 2004.

[34] P. Mittal and K. Gupta, "An integral involving generalized function of two variables," in Indian Acad. Sci., 1972, pp. 117-123.

[35] D. Yoon, "New expression for the SER of M-ary PSK," IEICE Transactions, vol. 88-B, no. 4, pp. 1672-1676, Apr. 2005.

[36] H. Soury, F. Yilmaz, and M.-S. Alouini, "Error rates of M-PAM and M-QAM in generalized fading and generalized Gaussian noise environments," IEEE Communications Letters, vol. 17, no. 10, pp. 19321935, October 2013, Extended version available as technical report at http://hdl.handle.net/10754/294163.

[37] M. A. Chaudhry and S. M. Zubair, On a Class of Incomplete Gamma Function with Applications. Boca Raton-London-Ney YorkWashington, D.C.: Chapman \& Hall/CRC, 2002.

[38] A. Kilbas and M. Saigo, H-Transforms : Theory and Applications (Analytical Method and Special Function), 1st ed. CRC Press, 2004.

[39] A. P. Prudnikov, Y. A. Brychkov, and O. I. Marichev, Integral and Series: Volume 3, More Special Functions. CRC Press Inc., 1990.

[40] Y. Jeong, H. Shin, and M. Z. Win, "H-transforms for wireless communication," IEEE Transactions on Information Theory, vol. 61, no. 7, pp. 3773-3809, July 2015.

[41] F. Yilmaz and M.-S. Alouini, "Product of the powers of generalized Nakagami-m variates and performance of cascaded fading channels," in Proc. of the IEEE Global Telecommunication Conference (GLOBECOM'2009), Honolulu, Hawai, USA, Dec 2009, pp. 1-8.

[42] K. Peppas, "A new formula for the average bit error probability of dualhop amplify-and-forward relaying systems over generalized shadowed fading channels," IEEE Wireless Commun. Lett., vol. 1, no. 2, pp. 8588, Apr 2012.

[43] L. Glasser, K. T. Kohl, C. Koutschan, V. H. Moll, and A. Straub, "The integrals in Gradshteyn and Ryzhik. Part 22: Bessel- $K$ functions." Scientia. Series A: Mathematical Sciences. New Series, vol. 22, pp. 129151, 2012.

[44] M. K. Simon and M.-S. Alouini, Digital Communication Over Fading Channels, 1st ed. New York: Wiley, 2005. 